Review Article

\title{
Thermosensitive Chitosan- $\beta$-Glycerophosphate Hydrogels as Targeted Drug Delivery Systems: An Overview on Preparation and Their Applications
}

\author{
Pouria Rahmanian-Devin, ${ }^{1,2}$ Vafa Baradaran Rahimi $\left(\mathbb{D},{ }^{2}\right.$ and Vahid Reza Askari $\mathbb{D D}^{2,3,4}$ \\ ${ }^{1}$ Department of Pharmaceutics, School of Pharmacy, Mashhad University of Medical Sciences, Mashhad, Iran \\ ${ }^{2}$ Applied Biomedical Research Center, Mashhad University of Medical Sciences, Mashhad, Iran \\ ${ }^{3}$ Department of Pharmaceutical Sciences in Persian Medicine, School of Persian and Complementary Medicine, \\ Mashhad University of Medical Sciences, Mashhad, Iran \\ ${ }^{4}$ Department of Persian Medicine, School of Persian and Complementary Medicine, Mashhad University of Medical Sciences, \\ Mashhad, Iran
}

Correspondence should be addressed to Vahid Reza Askari; askariv@mums.ac.ir

Received 1 November 2020; Revised 9 April 2021; Accepted 20 April 2021; Published 5 May 2021

Academic Editor: Abdeslam Jaafari

Copyright (C) 2021 Pouria Rahmanian-Devin et al. This is an open access article distributed under the Creative Commons Attribution License, which permits unrestricted use, distribution, and reproduction in any medium, provided the original work is properly cited.

\begin{abstract}
Today, with the advances in technology and science, more advanced drug delivery formulations are required. One of these new systems is an intelligent hydrogel. These systems are affected by the environment or conditions that become a gel, stay in the circumstance for a certain period, and slowly release the drug. As an advantage, only a lower dose of the drug is required, and it provides less toxicity and minor damage to other tissues. Hydrogels are of different types, including temperature-sensitive, $\mathrm{pH}-$ sensitive, ion change-sensitive, and magnetic field-sensitive. In this study, we investigated a kind of temperature-sensitive smart hydrogel, which has a liquid form at room temperature and becomes gel with increasing temperature. Chitosan- $\beta$-glycerophosphate hydrogels have been researched and used in many studies. This study investigates the various factors that influence the gelation mechanism, such as gel formation rates, temperature, $\mathrm{pH}$, time, and gel specificity. Hydrogels are used in many drug delivery systems and diseases, including nasal drug delivery, vaginal drug delivery, wound healing, peritoneal adhesion, ophthalmic drug delivery, tissue engineering, and peptide and protein delivery. Overall, the chitosan- $\beta$-glycerophosphate hydrogel is a suitable drug carrier for a wide range of drugs. It shows little toxicity to the body, is biodegradable, and is compatible with other organs. This system can be used in different conditions and different medication ways, such as oral, nasal, and injection.
\end{abstract}

\section{Introduction}

Today, with the advances in technology and science, new pharmaceutical formulations have been investigated. One of the new types of systems is smart hydrogels, which become gel based on environmental factors or specific conditions, stay in place for a certain period, and slowly release the drug. In these systems, a certain amount of the drug is delivered to the target site, and other tissues are less damaged. Hydrogels are of various types, including temperature-sensitive, $\mathrm{pH}$ sensitive, ion change-sensitive, and magnetic field-sensitive. In this study, a variety of temperature-sensitive hydrogel, chitosan- $\beta$-glycerophosphate, was investigated and evaluated. It has a liquid form at room temperature but becomes gel with an increase in temperature. This hydrogel can deliver a wide range of drugs. In this study, we investigate the various factors that influence the gelation mechanism $[1,2]$.

Hydrogels are three-dimensional hydrophilic polymers that can absorb large amounts of water [2] and are used for therapeutic applications since 1960. Hydrogels can provide networks physically or chemically. This network density controls the porous structure of the hydrogels. Hydrogels can be used for drug delivery and tissue engineering purposes because of their biocompatibility, high water content, 
low surface tension, and mechanical properties similar to those of hydrogels with body tissues. Physically networked hydrogels are soluble in water, which becomes gel due to changing environmental conditions such as temperature, $\mathrm{pH}$, light, electric field, and ionic strength. In fact, there is no covalent bond between the polymer chains of these hydrogels. In contrast, there is a covalent bond between the polymer chains of chemically networked hydrogels. Natural polymers, often called biopolymers, have long been used to develop hydrogels [3]. The highly porous structure hydrogels can be adjusted by controlling the density of cross-links in the gel and the amount of hydrogel bonding in the aqueous medium in which they are located. The hydrogel structure's porosity allows the drug to be loaded into the hydrogel and then released in a rate-dependent manner on the penetration coefficient of small or large molecules into the gel network [4].

Hydrogels have hydrophilic and hydrophobic portions that prevent the polymer from dissolving in water [5]. Hydrogels can entrap water molecules $(\geq 90 \% w / w)$ into their structure through their hydrophilic groups, such as the amine $\left(\mathrm{NH}_{2}\right)$, the carboxylic acid $(\mathrm{COOH})$, and sulfate $\left(\mathrm{SO}_{3} \mathrm{H}\right)$ [6]. Hydrogels can be swollen reversibly, and their swelling properties largely depend on their surroundings factors [7-9]. The temperature influences interactions between the hydrophilic and hydrophobic units with the water molecule. Therefore, these changes can increase the solubility of the cross-linked network. This factor is the leading cause of the solid phase change from liquid to solid [10]. The change in the equilibrium between hydrophilicity and hydrophobicity determines the cross-links' dissolution behaviour [11].

\section{Stimuli-Sensitive Hydrogels}

Smart hydrogels are used to release the drug in the body and deliver the protein drugs. The rate of drug release from this system can be modified with changing environmental conditions. Smart hydrogels can be controlled by different changes in their swelling behaviour and mechanical strength in response to various stimuli such as $\mathrm{pH}$, temperature, light, and electric or magnetic field. They are swollen or wrinkled with minor changes in environmental conditions. The water molecules interact with the hydrogel hydrophilic groups in the aquatic environment and form a regular arrangement around the hydrogel strands. When there is hydrogel swelling, it can be fragmented and dissolved if the network connections are degradable [12].

2.1.Temperature-Sensitive Hydrogels. Temperature-sensitive hydrogels are liquefied at room temperature but become gel at a physiological temperature or above that temperature. Chenit and Cho [13] showed that the mechanism of chitosan-glycerol phosphate hydrogel gelation depends on several factors, including the following:

(1) Hydrogen bonds between chitosan chains-electrostatic interactions and hydrophobic interactions have the main effect on gel formation
(2) The addition of chitosan increases the ionic strength, which reduces electrostatic repulsion between chitosan chains

(3) Electrostatic attraction occurs between the ammonium group's opposing forces in chitosan and the phosphate group in glycerolphosphate

Moreover, hydrophobic groups in glycerolphosphate improve hydrophobic interactions between chitosan molecules [13].

2.1.1. Classification of Temperature-Sensitive Hydrogels. Among the hydrogels, chitosan-based hydrogels are mostly considered for applications due to their biocompatibility and biodegradability, delivery of hydrophilic and hydrophobic drugs, relative $\mathrm{pH}$ stability, and easy formulation (Table 1) [26]. In this regard, several studies reported the applications. Bhattarai et al. [27] loaded PEG into chitosan to form a reversible hydrogel without binding. They developed hydrogels based on poly ethylene glycol-graft-chitosan (PEG-g-chitosan) without additional binders. Poly(ethylene glycol)-graft-chitosan was obtained by binding monohydroxy polyethene glycol to a chitosan scaffold.

By optimising the content and molecular weight of PEG in PEG-g-chitosan polymer, a temperature-dependent reversible phase transition is achieved at the normal room temperature $\left(22-25^{\circ} \mathrm{C}\right)$, which is converted to gel at the body temperature $\left(37^{\circ} \mathrm{C}\right)$. The phase change stage is directly related to the chitosan chains and the decrease in the polyethene glycol junctions' mobility at high temperatures. In one study, the release of bovine serum albumin from PEGchitosan hydrogels was assessed. It was observed after a sudden release of the drug at the beginning of the administration, and the residual amount of the drug was released within 70 hours [28]. Chitosan properties can be modified by incorporating hydroxy butyl groups in its main chain to improve its solubility, temperature sensitivity, and plasticity, and the gel is formed at body temperature [29]. The mixture of chitosan and gelatine can produce hydrogels for topical drug delivery and slow release of protein and peptide [30].

A new temperature-sensitive chitosan hydrogel system has been developed from a combination of chitosan and poly (vinyl alcohol) (PVA) used for protein transfer. This hydrogel is formed by hydrogen bonds between the chitosan and PVA chains, and the hydrophobic interactions of the chitosan chains [31]. Adding hydroxyapatite to the chitosanPVA hydrogel creates a composite that has high tensile strength. The hydrogel of chitosan and potassium hydrogen orthophosphate dipotassium hydrogen (orthophosphate (chitosan/DHO)) can be considered very effective as a controlled-release system in orthopaedic diseases [32]. For instance, doxorubicin (Dox)-loaded chitosan/DHO hydrogel was used clinically to evaluate its therapeutic effect on the orthopaedic osteosarcoma (OS) model. Significant reductions were observed in both primary and secondary osteosarcoma models, and the hydrogel system also reduced Dox-induced cardiac and skin toxicity in mice. Moreover, they evaluated the effect of Pigment epithelium-derived 
TABLE 1: Type of thermo-responsive hydrogels.

\begin{tabular}{|c|c|c|c|c|c|c|c|}
\hline & $\begin{array}{c}\text { Polymers } \\
\text { combined with }\end{array}$ & $\begin{array}{c}\text { Gelling } \\
\text { temperature } \\
{ }^{\circ} \mathrm{C} \\
\end{array}$ & $\begin{array}{l}\text { In the } \\
\text { market }\end{array}$ & Disadvantages & Advantages & Application & Reference \\
\hline \multicolumn{8}{|l|}{$\begin{array}{l}\text { 1. Cellulose } \\
\text { derivative }\end{array}$} \\
\hline 1.1 methylcellulose & $\begin{array}{l}\mathrm{N} \text {-isopropyl } \\
\text { acrylamide } \\
\text { (NiPAAM) }\end{array}$ & $60-80$ & & & & $\begin{array}{l}\text { Create a } \\
\text { bioactive } \\
\text { scaffold }\end{array}$ & {$[14]$} \\
\hline 1.2 chitosan & $\begin{array}{c}\text { PEG [15] } \\
\text { N-isopropyl } \\
\text { acrylamide } \\
\text { (NiPAAM) [16] } \\
\text { Glycerophosphate } \\
\text { [17] }\end{array}$ & 37 & $\begin{array}{c}\text { BST- } \\
\text { Gel }{ }^{\circledR} \\
{[18]}\end{array}$ & $\begin{array}{l}\text { The initial release of } \\
\text { the drug is high. } \\
\text { It always retains } \\
\text { some of the drugs. } \\
\text { It has a low rate of } \\
\text { destruction }\end{array}$ & $\begin{array}{c}\text { Forms a reversible } \\
\text { gel that gels when } \\
\text { placed in the body. } \\
\text { It can release the } \\
\text { drug for a long } \\
\text { time. } \\
\text { Biodegradable and } \\
\text { good } \\
\text { biocompatibility }\end{array}$ & $\begin{array}{l}\text { Cell delivery } \\
\text { Drug delivery } \\
\text { Implant } \\
\text { Similar } \\
\text { structure to } \\
\text { extracellular } \\
\text { matrix }\end{array}$ & {$[17]$} \\
\hline 1.3 Dextran & $\begin{array}{l}\text { N-isopropyl } \\
\text { acrylamide } \\
\text { (NiPAAM) }\end{array}$ & $32-37$ & & $\begin{array}{c}\text { At a temperature of } \\
37 \text { degrees, its } \\
\text { degradation rate } \\
\text { decreases. } \\
\text { Drug release } \\
\text { depends on various } \\
\text { factors such as pH } \\
\text { and electrolyte }\end{array}$ & $\begin{array}{l}\text { It can create a } \\
\text { sustained release } \\
\text { formulation }\end{array}$ & Drug delivery & [19] \\
\hline \multicolumn{8}{|l|}{ 2. Proteins } \\
\hline 2.1 Gelatine & $\begin{array}{c}\text { Poly(ethylene } \\
\text { glycol)-Poly (D, L- } \\
\text { lactic) } \\
\text { (mPEG-DLLA) } \\
{[20]}\end{array}$ & Below 25 & & $\begin{array}{l}\text { Improper gelling } \\
\text { and adhesion } \\
\text { properties } \\
\text { The initial release of } \\
\text { the drug is high }\end{array}$ & $\begin{array}{c}\text { Slow-release } \\
\text { profile }\end{array}$ & $\begin{array}{l}\text { Drug release } \\
\text { kinetic with } \\
\text { gentamycin } \\
\quad \text { sulfate }\end{array}$ & {$[21]$} \\
\hline \multicolumn{8}{|l|}{ Other polymers } \\
\hline $\begin{array}{l}\text { 3. N- } \\
\text { isopropylacrylamide }\end{array}$ & & 32 & & $\begin{array}{l}\text { Nonbiodegradable } \\
\text { The gelling } \\
\text { temperature } \\
\text { depends on the } \mathrm{pH} \\
\text { and electrolytes of } \\
\text { the environment. } \\
\text { Swelling was lower } \\
\text { Initial burst release }\end{array}$ & $\begin{array}{l}\text { Preparation of } \\
\text { implants } \\
\text { Long-term drug } \\
\text { release }\end{array}$ & $\begin{array}{c}\text { Drug delivery } \\
\text { Cell } \\
\text { encapsulation } \\
\text { Cell culture } \\
\text { Biomedical } \\
\text { engineering } \\
\text { application }\end{array}$ & {$[22]$} \\
\hline $\begin{array}{l}\text { 4. } \mathrm{PEO} / \mathrm{PPO} \\
\text { Poloxamer }{ }^{\circledR}\end{array}$ & $\begin{array}{c}\text { Poly (ether- } \\
\text { carbonate) }\end{array}$ & 37 & $\begin{array}{l}\text { LeGoo } \\
{ }^{\circledR}[23]\end{array}$ & $\begin{array}{c}\text { Lower stiffness than } \\
\text { other hydrogels } \\
\text { After one week } \\
\text { degraded }\end{array}$ & $\begin{array}{l}\text { Biocompatible } \\
\text { High viscosity }\end{array}$ & $\begin{array}{l}\text { Drug and gene } \\
\text { delivery } \\
\text { Tissue } \\
\text { adhesive } \\
\text { Burn wound } \\
\text { covering }\end{array}$ & {$[24,25]$} \\
\hline
\end{tabular}

factor delivery with hydrogel on the inhibition of osteosarcoma. The results showed that a combination of plasmid treatment and chemotherapy, combined with the use of chitosan/DHO hydrogels, resulted in significant inhibition of tumour cell growth without adverse effects [32].

2.1.2. Positive and Negative Temperature-Sensitive Hydrogels. Positive temperature-sensitive hydrogels' solubility in water is increased by increasing temperature. Inversely, negative temperature-sensitive hydrogels are shrunk by increasing temperature. At low temperatures, hydrogen bonds are formed between hydrophilic groups of polymer chains and water molecules. As the temperature increases, the hydrophobic interactions between the hydrophobic groups become more vigorous, while the hydrogen bonds become weaker [33]. Changing the equilibrium between hydrophilicity and hydrophobicity determines the dissolution behaviour of the cross-links [11]. The hydrogel is soluble below the Lower Critical Solution Temperature (LCST), while when exposed to the high LCST, it becomes hydrophobic and insoluble, and the gel is formed. The LCST can be modified by changing the hydrophilicity/hydrophobicity ratio. Another hydrogels group is liquefied at temperatures above the Upper Critical Solution Temperature (UCST) and gel at temperatures below this temperature [34]. 
2.2. Chitosan. Chitosan is a biopolymer that has been extensively studied recently [35]. It is a linear polysaccharide formed by the deacetylation of chitin. Chitin is one of the significant components of the outer skeleton of crabs, insects, and the fungal wall [27]. Chitosan is composed of $\mathrm{D}$-glucosamine and n-acetyl-D-glucosamine units, which are randomly interconnected. Chitosan is approved by the US Food and Drug Administration (FDA) [36] for its nontoxicity, biodegradability, biocompatibility, and bioavailability, and has been widely used in engineering and medical fields [26]. Indeed, its unique properties, such as biodegradability, nontoxicity, antibacterial effects, biocompatibility, and adhesion mucus, have led to its wide use as a biomaterial for tissue engineering applications and drug delivery $[37,38]$. The results of various studies have shown that chitosan had appropriate wound-healing effects and reduced inflammation. Also, biological properties such as bio-adhesion, anti-cancer, antimicrobial, inflammatory and nociceptive, antioxidant, blood coagulant, and cholesterollowering agents make it distinct from other biopolymers $[39,40]$. It has been used for over a decade as a safe compound in drug formulation and also, because of its adhesion properties, can be used as an effective material in bonding hard and soft tissues $[34,41]$. Chitosan films with a low degree of deacetylation are very suitable for wound healing. They attach to the surface of the tissue and increase the number of keratinocytes, thereby producing new tissue [42]. Chitosan also has an antiacid activity, which prevents the reduction of the effect of drugs on the stomach [43]. Chitosan-based polymer systems are also used to deliver and release proteins/peptides, growth factors, painkillers, antibiotics, antibiotics, cancer drugs, and inflammation, and to treat gene deficiencies. [44]. Chitosan solubility in weak acids is the best justification for its popularity with chitin due to the presence of the first type of amine groups. Its biodegradability by several enzymes and the formation of gels at low $\mathrm{pH}$ has made it a unique biopolymer [45].

One of the most recent chitosan applications in the design of slow-release drug delivery systems is the use of chitosan-glycerol phosphate temperature-sensitive solution as a biodegradable gel-forming system at the injection site. Ohya et al. [46] first reported on the use of chitosan nanoparticles in drug applications. They prepared water in oil emulsion of chitosan and then added glutaraldehyde into the emulsion for forming cross-links between the citizen amine groups. Finally, the nanoparticles were created, and 5fluorouracil, an anticancer drug, was loaded on them. Due to the 5-FU derivatives having an amine-terminal group in their formulation, glutaraldehyde's addition forms similar linkages to the chitosan chain between them. As a result, the drug binds to the polymer chain and becomes completely immobilised. One study demonstrated that it is possible to synthesise stable chitosan nanoparticles capable of holding and delivering the drug [46].

As mentioned, chitosan is soluble in water and has a cationic nature. This feature enables chitosan to interact with polymers or macromolecules that are anionic in nature and have certain polyanions in the aqua environment. These interactions are used to make chitosan nanoparticles. The ability to form gels in contact with anionic groups is an interesting feature of chitosan called ionic gelation (Ionotropic Gelation). This gel formation process is due to the formation of intrachain and interchain cross-links caused by anionic groups' presence. The most well-known reaction is the chitosan reaction with triphosphate groups (TPP) [47]. Anionic TPP as the cross-linking agent for the preparation of chitosan nanohydrogels is one of the most well-known methods for preparing chitosan nanohydrogels. In one study, insulin-loaded chitosan nanoparticles were prepared by mixing insulin with the TPP solution and then slowly adding this compound to the chitosan solution while stirring. By this method, the insulin loading rate was optimised and reached 55\% efficiency [48]. Many studies proved that the bioavailability of peptides and proteins is improved by this method for oral delivery. The study also claimed that the bio-stickiness of chitosan enhances the adhesion of drugs to the gut. Zhou et al. [49] studied different formulations of chitosan-TPP nanoparticles prepared by the ion gelation method. TEM images showed that the shape of the particles was spherical, and the diameter of the resulting particles was 20 to $200 \mathrm{~nm}$ [50]. Chitosan has a remarkable ability to adhere to mucosal surfaces in the body. In fact, this feature has drawn attention to this polymer in mucosal drug delivery. This unique ability of chitosan has been used to open tight junctions between mucosal cells $[51,52]$.

2.3. Temperature-Sensitive Chitosan Hydrogels. Chitosan is dissolved in dilute organic acid solutions but is insoluble in high concentrations of hydrogen ions at $\mathrm{pH} 6.5$ and is precipitated as a gel-like compound. Chitosan is positively charged by amine groups, making it suitable for binding to negatively charged molecules. However, it has disadvantages such as low mechanical strength and low-temperature response rate; it must be combined with other gelling agents to improve its properties [38, 53]. Using glycerolphosphate salts (possessing a single anionic head) without chemical modification or cross-linking, the $\mathrm{pH}$-dependent gelation properties can be converted to temperature-sensitive gelation properties [54]. In the year 2000, Chenite was the first to design the temperature-sensitive chitosan hydrogels drug delivery system using chitosan and $\beta$-glycerol phosphate [17]. This new system can remain in the liquid state at room temperature, while becoming gel with increasing temperature above the physiological temperature $\left(37^{\circ} \mathrm{C}\right)$. Phosphate salts cause a particular behaviour in chitosan solutions, thereby allowing these solutions to remain soluble in the physiological $\mathrm{pH}$ range ( $\mathrm{pH} 7$ ), and they will be gel only at body temperature. When the liquid solution of chitosanglycerol phosphate, containing the drug, enters the body through a syringe injection, it becomes a water-insoluble gel at $37^{\circ} \mathrm{C}$. The entrapped drug particles between the hydrogel chains will be gradually released $[38,53]$.

2.4. $\beta$-Glycerophosphate. Beta $(\beta)$-glycerol phosphate is an organic compound found naturally in the body. It is commonly used as a phosphate source in treating phosphate metabolism imbalances and has been approved by the FDA 
for intravenous administration. $\beta$-glycerophosphate is considered an osteogenic factor in the culture medium of human bone marrow stem cells, which leads to the differentiation of bone marrow cells to bone cells [55]. It is also used as a serinethreonine phosphatase inhibitor. This compound is often used together with other inhibitors to inhibit a wide range of phosphatases and proteases. Moreover, $\beta$-glycerophosphate is used as a catalyst in chitosan temperature-sensitive hydrogels and converts fluid to gel phase in chitosan solutions at $\mathrm{pH}$ and physiological temperatures. Chitosan hydrogels have been prepared in various formulations, including liquid gels, granules, films, tablets, capsules, microspheres, microparticles, and nanofibers $[47,56]$. In each preparation, polymer bonding is performed using noncovalent auxiliary bonds, such as hydrogen, ionic, hydrophobic and physical bonds, or by covalent chemical cross-linking [41]. Related physical joints can often be obtained by mixing the constituents of the gel under appropriate conditions. Besides, gelation occurs without the need for a toxic binding molecule to the body. In this way, it will have healthy clinical applications forever [49]. The $\alpha / \beta$-glycerophosphate is a mixture of $\alpha$-glycerophosphate and $\beta$-glycerophosphate. $\alpha$-glycerophosphate has a linear chain structure that easily intercalates between chitosan molecules, whereas the hydrophobic force between chitosan molecules is more easily formed. This hydrophobic force causes the water molecules to lie in the chitosan chains' chimney and develop a regular arrangement around them, resulting in the chitosan being dissolved in water. Therefore, $\alpha / \beta$-glycerophosphate may have a similar gelation capacity compared with $\beta$-glycerophosphate [57]. In this regard, Wu et al. showed that by combining chitosan and $\alpha / \beta$-glycerophosphate and chitosan and $\beta$-glycerophosphate, temperature-sensitive hydrogels could be prepared [57].

\subsection{The Mechanism of Chitosan- $\beta$-Glycerophosphate Hydrogel} and Factors Influencing Gelation. The polyols surround the chitosan and provide a protective and hydro-resistant layer around the chitosan chains through weak intermolecular interactions such as hydrogen bonding. Increasing the temperature eliminates this polyol layer and allows the polymer to be in equilibrium through stronger hydrophobic bonds, thereby generating gels (Figure 1) [1]. The molecular mechanism of gelation may be due to multiple interactions between chitosan, $\beta$-glycerophosphate, and water [58]. Effective interactions responsible for converting liquid to gel are: (1) An essential role of $\beta$-glycerophosphate salts as a reducing electrostatic repulsion agent is to increase the hydrogen bonding between the chitosan chains; (2) Electrostatic attractions of chitosan- $\beta$-glycerophosphate through ammonium groups and phosphate groups; (3) Chitosan-chitosan hydrophobic interactions are enhanced by the structural role of $\beta$-glycerolphosphate on water, and the concentration of chitosan and $\beta$-glycerophosphate influences the soluble behaviour of chitosan and $\beta$-glycerophosphate hydrogels.

Due to the phosphate groups' neutralising effect, increasing $\beta$-glycerophosphate concentration slightly increases the chitosan solution's $\mathrm{pH}$. Increasing $\mathrm{pH}$ and increasing polymer concentration consume $\mathrm{H}^{+}$ions in the solution through the amine groups. The gelation temperature is gradually decreased with increasing $\beta$-glycerophosphate concentration and chitosan concentration. However, a synergistic effect at high $\beta$-glycerophosphate and high chitosan concentrations result in a sudden drop in gelation temperature and phase transition due to the gels' concentration and heat boundary. Increasing the temperature does not affect the $\mathrm{pH}$ of chitosan-glycerophosphate hydrogel system and its conductivity. The results show that the ionic interactions are decreased at high temperatures by decreasing the ratio of $\mathrm{NH} 3$ ion groups to chitosan and phosphate group to beta glycerolphosphate [59]. In fact, $\beta$-glycerophosphate plays three major roles in the system: (1) Increases the $\mathrm{pH}$ in the physiological range (from 7 to 7.4); (2) Prevents sudden deposition or rapid gelation; (3) Creates some control over the formation of the gel by increasing the temperature.

The temperature of liquid-to-gel conversion is sensitive to the environment's $\mathrm{pH}$ and the gelation time is temperature-dependent. Depending on the material's gelation behaviour, the hydrogels' rheological properties follow three types: (1) A liquid-like behaviour at low temperature; (2) A fast gelation process at a temperature near the gelation point; (3) A slow gelation process at high temperature. When the temperature is increased, the water molecules are exited from the chitosan chains, and the heat accelerates the proton transfer from the protonated amino acids to glycerophosphate. As a result, the formation of hydrophobic interactions between Chitosan chains is facilitated. In the study of rheological behaviour, the system shows the lowest level of contact obtained by the Ostwald ripening process. Also, the molecular weight and the degree of citizen distillation affect the chelation process $[54,59]$. It has been observed that the gelating point is $33^{\circ} \mathrm{C}$ for the lower molecular weight chitosan sample, which is $2^{\circ} \mathrm{C}$ higher than the high molecular weight chitosan sample. It means that hydrophobic interactions occur in lower molecular weight chitosan samples at higher temperatures. It can be the result of more cross-links and shorter hydrophobic groups. Such an observation can be attributed to the low molecular weight and low viscosity of the sample, thereby increasing the possibility of accumulation and penetration of hydrophobic groups. The lower molecular weight facilitates the accumulation and penetration of hydrophobic groups. Therefore, gelation occurs at a lower temperature.

The deacetylation of chitin chains to get more chitosan occurs at amorphous sites. Thus, the type of hydrophobic and hydrophilic joints was the main result of this deacetylation. Therefore, deacetylation affects the gelation process by simulating the hydrophobic properties of chitosan. Chitosan can be considered a bond of two polymers consisting of deacetylated and acetylated units. Moreover, this gelation can occur depending on the degree of chitosan deacetylation at lower temperatures [60]. In this way, the higher degree of chitosan deacetylation results in an acceleration in the gelation speed. It may be due to the increased bond density and cross-linkages between the 


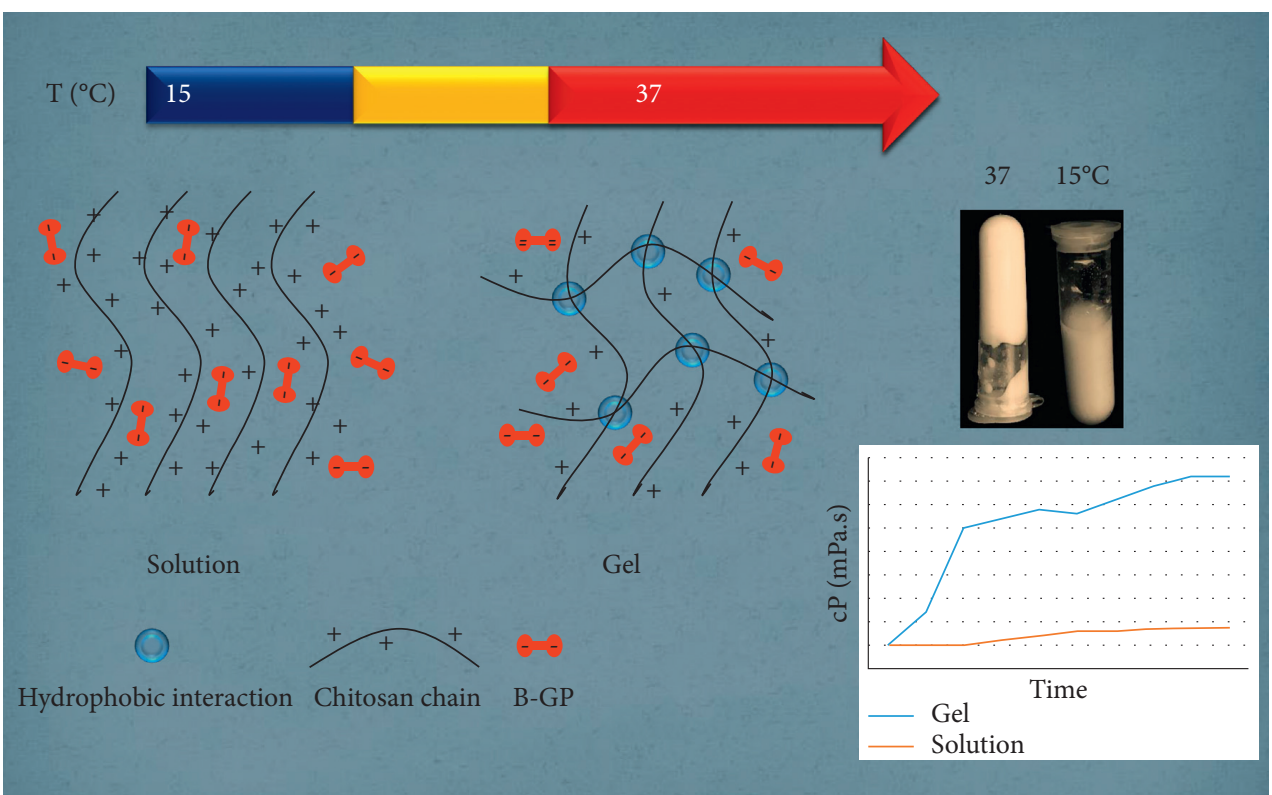

(a)

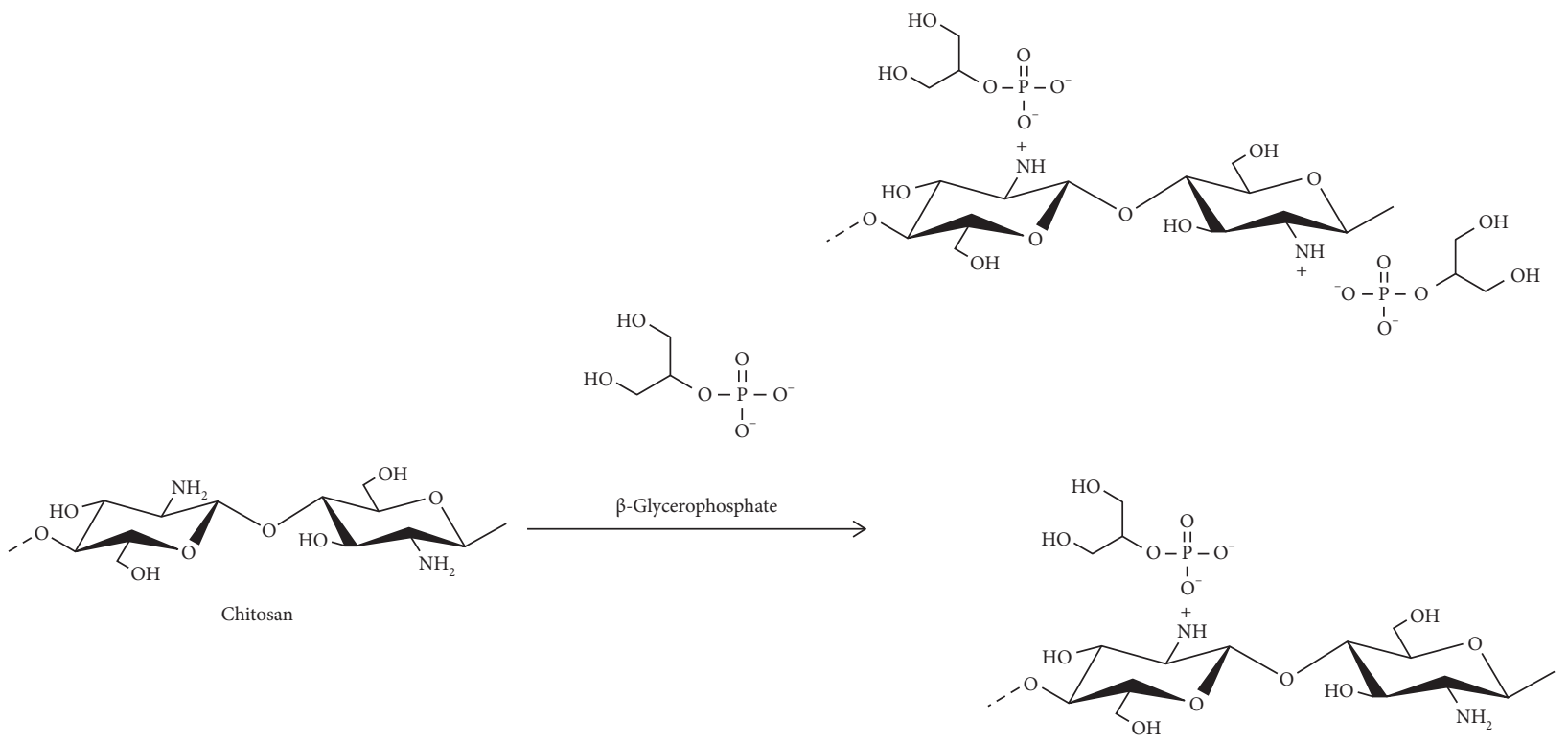

(b)

Figure 1: In an acidic environment, chitosan is positively charged (a). Because of the similar groups on the chitosan chain in an acidic environment, the two chains move away from one another, forming an electrostatic repulsion force between the chitosan chains, the water molecules being positioned between the chitosan chains and forming a regular arrangement (b). Adding beta-glycerophosphate, the negative charge of the positively charged chitosan phosphate group forms weak bonds. These weak interactions enhance the water arrangement around the chitosan chains. As a result, chitosan dissolves at physiological $\mathrm{pH}$. Nevertheless, with increasing temperature, the regular arrangement of water molecules around the chitosan chains becomes unstable, the polymer-polymer interactions overwhelm the solvent-polymer interactions, and the chitosan precipitates at high temperature in the form of gels [54].

phosphate groups of the glycerophosphate molecule and the ammonium groups of chitosan.

The gelation time is decreased with increasing $\beta$-glycerophosphate content, and gelation occurs at lower temperatures and at a lower time. By increasing the chitosan concentration, the gelation rate is increased, but the gelation occurs at a higher temperature $(2 \% w / v$ is the best concentration). However, by reducing the molecular weight of chitosan, the gelation time is increased. On the other hand, gelation temperature can be decreased and varied in the range of $37-32^{\circ} \mathrm{C}$ by increasing chitosan deacetylation degree, increasing $\beta$-glycerophosphate concentration, increasing $\mathrm{pH}$ (6.8-6.8), or reducing chitosan molecular weight $[61,62]$.

Preparing the temperature-sensitive hydrogels is dependent on the hydrophobic-hydrophilic reactions of the 
chain molecules. The results show that the gelation time, structure, swelling rate, and degradation rate in the in vivo and in vitro environments depend on the cross-links and the hydrogel structure. It has been observed that the gelation and swelling times depend on the binding, overlap, and adsorption of the polymer chains at different $\mathrm{pH}$ and temperatures. Finally, it can be said that in aqueous solutions of chitosan-glycerol phosphate, (1) the electrostatic attractor amine and phosphate groups stabilise the solubility at ambient temperature and (2) hydrogen bonds between the chains along with the chitosan-chitosan hydrophobic interactions cause the gel formation at body temperature (Figure 1) [63]. Table 2 shows several factors affecting gelation time.

\subsection{Chitosan-Glycerophosphate Hydrogel Derivatives.} Chitosan and its derivatives become thermally sensitive by adding glycerophosphate to a $2 \% \mathrm{w} / v$ chitosan solution (Figure 2) $[69,70]$. The rate of degradation of chitosan-based hydrogels largely depends on the cross-linking of another class of gel-forming compounds such as gelatine and genipine $[71,72]$. The drug release profile of chitosan hydrogels is affected by the degree of hydrophobicity of the drug. Compounds such as latanoprost and ferulic acid, which have low solubility in water $(\leq 1 \mathrm{mg} / \mathrm{mL})$, are slowly released from hydrogels (28\% in the first 24 hours). But, water-soluble compounds such as timolol maleate $(2.74 \mathrm{mg} / \mathrm{mL})$ have a high release rate (almost $80 \%$ of the drug in the first 24 hours). Chitosan hydrogels with $100 \%$ gelatine or crosslinked with a secondary linker, such as genipine or hydroxyapatite, will not have a $100 \%$ release in the long run. These secondary cross-links cause slower drug release due to the creation of smaller pores within the hydrogel networks $[70,73]$.

Chitosan- $\beta$-glycerophosphate hydrogels have no sufficient mechanical properties for some drug delivery systems, including for cell loading. Due to its biocompatibility properties, degradability, and ability to support cell growth, collagen and its derivatives, including gelatine, were selected as the first polymer to modify the properties of hydrogels. In one study, the addition of $2 \%$ gelatine to chitosan/glycerophosphate solution reduced the gelation temperature from $36^{\circ} \mathrm{C}$ to $31^{\circ} \mathrm{C}$ and reduced the gelation time at $37^{\circ} \mathrm{C}$ from 10 minutes to 2.3 seconds [1]. The mechanical strength of hydrogels is also improved with increasing gelatine concentration. Furthermore, chitosan/gelatine/glycerophosphate hydrogels have all of the biocompatibility and biodegradability properties found in chitosan/glycerophosphate hydrogels. Nevertheless, the addition of gelatine reduces the solubility of the solution at room temperature. Even at room temperature, the gel formulation can be done, but this can be reduced by lowering the gelatine concentration [74]. Sodium hydrogen carbonate $\left(\mathrm{NaHCO}_{3}\right)$ is applied to produce high-strength chitosan- $\beta$-glycerophosphate hydrogel. Hydrogels that contain large amounts of $\mathrm{NaHCO}_{3}$ have shorter gelation time and are more resistant in compression. These hydrogels create a highly porous structure, which provides suitable conditions for cell growth and has good biocompatibility at low $\beta$-glycerophosphate concentrations. Therefore, adding appropriate amounts of $\mathrm{NaHCO}_{3}$ to the chitosan $\beta$-glycerophosphate hydrogel can improve its properties [75]. Wu and his colleagues developed a temperature- and $\mathrm{pH}$-sensitive hydrogel from the combination of chitosan and quaternized chitosan (HTCC) containing $\alpha, \beta$-GP (CS-HTCC/GP), which investigated doxorubicin's release behaviour in various modes [57, 76]. Chitosan-HTICC-GP hydrogels are also used for nasal drug delivery. Placing insulin on the hydrogel and using it through the nose reduced blood sugar by $50-50 \%$ for $4-5$ hours. In addition, this hydrogel is highly biocompatible and easily degraded and excreted by the body [38].

\section{Advantages of Temperature-Dependent Chitosan- $\beta$-Glycerophosphate Hydrogels}

3.1. Biocompatibility and Biodegradability. One of the major advantages of chitosan hydrogel systems, unlike other drug delivery systems such as implants, is the degradable system that eliminates after a while and does not require surgery to remove the carrier. Chitosan is attacked and destroyed by the body's hydrolytic enzymes such as lysozyme [27, 77]. Lysozyme in various fluids and tissues in the human body are found, with concentrations ranging from 4 to $13 \mathrm{mg} / \mathrm{l}$. The results show that the rate of degradation of chitosan hydrogels is correlated with the degree of chitosan deacetylation. If the chitosan deacetylation degree is high, the rate of hydrogel degradation is lower [78]. By increasing the concentration of chitosan, the rate of degradation is decreased. Hydrogel degradation by lysozyme begins at its surface and gradually penetrates it. Continuous degradation of hydrogels occurs through the hydrolysis of acetyl groups. The hydrogel network's porous structure is maintained for seven days in vitro while significantly reduced and degraded in vivo. Similar studies have shown that after intramuscular injection of the hydrogel, it begins to degrade after eight weeks and is completely degraded after 12 weeks [79]. The destruction of hydrogels in the body should be considered on the drug pharmacokinetics because it may alter the rate of drug release in the long term [1].

3.2. Porous Structure and Heterogeneous Morphology. Ahmadi and Borujen revealed that chitosan-glycerolphosphate hydrogel could stimulate mesenchymal stem cell (MSC) proliferation at specific concentrations, indicating that it would be a suitable carrier for cell encapsulation and tissue engineering [80]. The morphology of chitosan glycerolphosphate was examined by laser scanning confocal microscopy (LSCM), and the results showed that the hydrogel had a completely heterogeneous morphology [81].

3.3. Controlled Release and Sustained Release. Kempe et al. found that insulin in the aqueous medium had its original spin-labeled hydrogel and was naturally released from the formulation [10]. Besides, they claimed that insulin is released into the chitosan hydrogel matrix by diffusion and 
TABLE 2: Factors influencing gelation, chitosan- $\beta$-glycerophosphate.

\begin{tabular}{lcccccccc}
\hline MW & Con & DDA $\%$ & Time $(\mathrm{min})$ & $\mathrm{pH}$ & $\mathrm{GP}$ & $T\left({ }^{\circ} \mathrm{C}\right)$ & Drug & Reference \\
\hline $310-375$ & $0.8 \mathrm{~g}$ & 103 & 5 & 7 & $2 \mathrm{~g}$ & 37 & Aspirin & {$[64]$} \\
62 & $0.1 \% w / w$ & 82 & 1.6 & 7.4 & $12 \%$ & 30 & Mitomycin-c & {$[65]$} \\
124 & $0.1 \% w / w$ & 72 & 1.4 & 7.4 & $12 \%$ & 29.8 & Mitomycin-c & {$[65]$} \\
370 & $0.1 \% w / w$ & 71 & 1 & 7.4 & $12 \%$ & 29.6 & Mitomycin-c & {$[65]$} \\
650 & $20 \mathrm{mg} / \mathrm{mL}$ & 85 & 10 & 7.35 & $3 \%$ & 37 & Curcumin \\
200 & $1.8 \% w / w$ & 91 & 12 & 6.88 & $9 w / w$ & 37 & Docetaxel & {$[66]$} \\
200 & $1.8 \% w / w$ & 91 & 5 & 6.97 & $12 w / w$ & 37 & Docetaxel \\
200 & $1.4 \% w / w$ & 91 & $>120$ & 6.75 & $6 w / w$ & 37 & Docetaxel & {$[67]$} \\
200 & $1.4 \% w / w$ & 91 & 61 & 6.83 & $9 \% w / w$ & 37 & Docetaxel \\
306.12 & $2 \% \% w / v$ & 95 & 2 & 7.63 & $14 \% w / v$ & 37 & Insulin \\
306.12 & $2 \% \% w / v$ & 95 & 3 & 7.52 & $12 \% w / v$ & 37 & Insulin \\
306.12 & $2 \% \% w / v$ & 95 & 5 & 7.45 & $10 \% w / v$ & 37 & Insulin \\
306.12 & $2 \% \% w / v$ & 95 & 14 & 6.91 & $5 \% w / v$ & 37 & Insulin & {$[68]$} \\
\hline
\end{tabular}

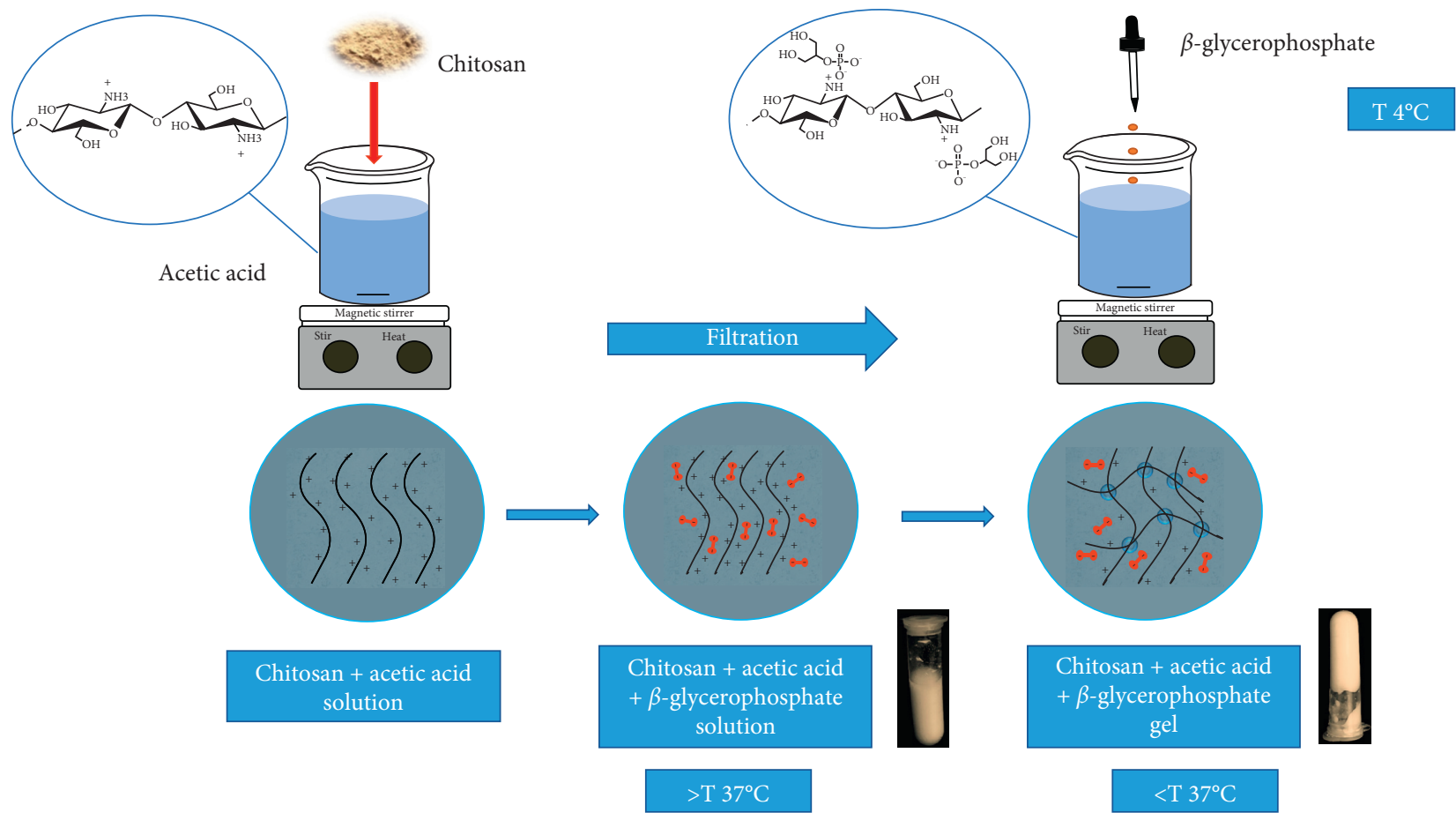

FIGURE 2: The schematic figure for preparing the chitosan-based hydrogel.

that the released drug increases with increasing glycerolphosphate ratio. Observations show that the drug release is slower when added to the chitosan solution alone than when the drug is added directly to the chitosan solution combined with glycerol-phosphate, in which chitosan-glycerol phosphate hydrogel can take the drug for several days. It has also been observed that the release of hydrophobic drugs such as Adriamycin ${ }^{\circledR}$ occurs less rapidly than hydrophilic drugs such as 6-mercaptopurin from chitosan glycerolphosphate hydrogel [82].

3.4. Drug Targeting. Hydrophobic drugs are first inserted into the liposome and then transferred to chitosan glycerol hydrogels to achieve a slow release. It was observed that the administration of in situ chitosan glycerolphosphate hydrogel containing paclitaxel prevented the recurrence of the tumour [83]. Chitosan glycerolphosphate hydrogel has also been used to transport cells into cartilage tissue for its repair [65]. In a study, ellagic acid was firstly loaded into PLGA and subsequently embedded in chitosan-glycerolphosphate hydrogel to improve its bioavailability and slow its excretion. As result, it was found that ellagic acid had a slower release and more appropriate bioavailability [84]. Moreover, Han et al. reported that hydrogel chitosan glycerolphosphate combined with chemotherapy drugs and viral vaccines had synergistic antitumour effects against the tumour [85]. In vitro studies of hydrogel chitosan glycerolphosphate containing Ellagic acid for brain cancer have been studied [26]. Recently, the use of hydrogel polymers similar to chitosan glycerolphosphate and elastin in bone tissue engineering has expanded and improved the 
mechanical properties of tissue under physiological conditions [8].

\section{Method for Preparation of Temperature- Sensitive Hydrogel Chitosan- Glycerophosphate}

The hydrogel can absorb much water as an effective drug delivery system that creates a porous structure $[27,86]$. Chitosan powder with a deacetylation degree more significant than $85 \%$ and a molecular weight of more than $650 \mathrm{kD}$ is commonly used to prepare hydrogels (Figure 2). The method is described as follows [66]. First, we dissolve a certain amount of chitosan in a $0.1 \mathrm{M}$ acetic acid solution and vigorously stir for $1 \mathrm{~h}$. We must remove the precipitated chitosan by filtration, then cool and place it at $4^{\circ} \mathrm{C}$ for storage. The prescribed amount of active ingredient was dispersed in $1 \mathrm{~mL}$ distilled water and treated by ultrasonication for $1 \mathrm{~h}$. Dissolve $300 \mathrm{mg}$ of glycerolphosphate in $1 \mathrm{~mL}$ of distilled water and pass the resulting solution through a $0.22 \mu \mathrm{m}$ filter. In the second step, $1 \mathrm{~mL}$ of active ingredient was added into the chitosan solution and stirred for $1 \mathrm{~h}$ until well dispersion. If the hydrogel must be sterile, then autoclave all solutions separately at $121^{\circ} \mathrm{C}$ for 15 minutes, cool to room temperature and store at $4^{\circ} \mathrm{C}$, and perform the rest of the steps in a sterile place (Like a laminar flow hood). Finally, the glycerophosphate solution was added dropwise to the active ingredient/chitosan mixed solution under constant stirring at low temperature. As the control group, the active ingredient/chitosan hydrogel was prepared following the same steps above, except that the active ingredient dispersion was replaced by $1 \mathrm{~mL}$ distilled water. Store it in the fridge. To investigate the morphology of the hydrogel, we use an SEM microscope. Put the hydrogel formulation at $37^{\circ} \mathrm{C}$ for 3 hours, then freeze it. Place the lyophilised powder at the desired location and coat the surface with a thin layer of gold [66].

\section{Sterilisation}

Injectable medications must be sterile and free of pathogens before usage. The final sterilisation process requires high temperature, such as steam or high heat, which cannot be used because of the hydrogel's thermosensitive property and the irreversibility of the chitosan-glycerophosphate hydrogels is impaired [87]. Therefore, gamma irradiation is one way of ultimate sterilisation, which reduces the hydrogel formulation's viscosity even at temperatures below $80^{\circ} \mathrm{C}$ due to the polymer chains' degradation [88]. On the other hand, sterilising the product using $0.2-\mu \mathrm{m}$ filters can be complicated depending on the viscosity of the solution or may not be possible if the drug or cells are dispersed in the solution [1]. In general, chitosan and $\beta$-glycerophosphate solutions are sterilised separately and mixed in aseptic conditions. Sterilising chitosan solution with steam before adding glycerophosphate is one of the common ways. However, this method directly damages the polymer and reduces its porosity and affects its viscosity [89]. Chitosan powder or solutions can be sterilised by ultraviolet, gamma-ray, or beta radiation, but these methods may damage the polymer structure and usually are not considered suitable. The best way to sterilise chitosan powder using water vapour seems to maintain the chitosan's molecular weight and then dissolve the chitosan in the acidic solvent under sterile conditions [90].

\section{Applications of Temperature-Sensitive Chitosan-Glycerophosphate Hydrogels}

Hydrogels are widely used to control and slow down the release and prolong drug delivery. Nowadays, It has several applications in tissue engineering and wound repair (Table 2 and Figure 3) [82]. Subcutaneous or intramuscular injection of the formulation can increase the release time from one day to one month, depending on the concentrations of chitosan and glycerophosphate, and the properties of the loaded drug. Several drugs have been studied with this method, such as aspirin [62], risperidone [91], insulin [53], methylprednisolone [92], docetaxel [67], venlafaxine $[66,93]$, doxorubicin $[66,94,95]$, curcumin [66], and some antibiotics, such as mitomycin [65], as carriers for tissue engineering and cell encapsulation [96] and protein and peptide [97], such as erythropoietin [64, 98] and nerve growth factor [71].

\subsection{Drug Delivery System}

6.1.1. Nasal Delivery. Temperature-sensitive hydrogels are an excellent option for nasal drug delivery. They are liquid at room temperature, easily dispersed in the nasal cavity during use, and then transformed into a gel at body temperature, remaining on-site for a long time, improving bioavailability and drug absorption [99, 100]. Chitosan is a biocompatible, biodegradable, and nontoxic polymer widely used as an effective carrier for enhancing drug absorption through the nasal passage. Chitosan binds weakly to the sialic acid present in the nasal mucosa, thereby creating a gap between the tight epithelial junctions and opening the drug delivery pathway, and increases the absorption of drugs. Moreover, chitosan also acts as a mucosal adhesive compound, increasing drug retention duration on the mucosa, increasing its bioavailability and uptake $[101,102]$. Further compounds, such as polyethene glycol and hydroxypropyl methylcellulose, are added to the formulation to improve the temperature-sensitive and adhesive properties of chitosan hydrogels [76]. However, these auxiliary interactions can irritate the nasal mucosa [103]. On the other hand, drug and carrier interactions lead to drug crystallisation inhibition and improve drug solubility [104]. Molecular interactions, especially hydrogen bonds between polymer chains and drug molecules, play a major role in drug efficacy [103].

Moreover, thermosensitive hydrogel formulations of chitosan are used to transport biological substances such as genes and proteins. However, there are limiting factors as well; for example, chitosan is soluble and can release hydrogen only in an acidic environment [105]. Substitution of trimethyl chitosan chloride derivatives instead of chitosan in 


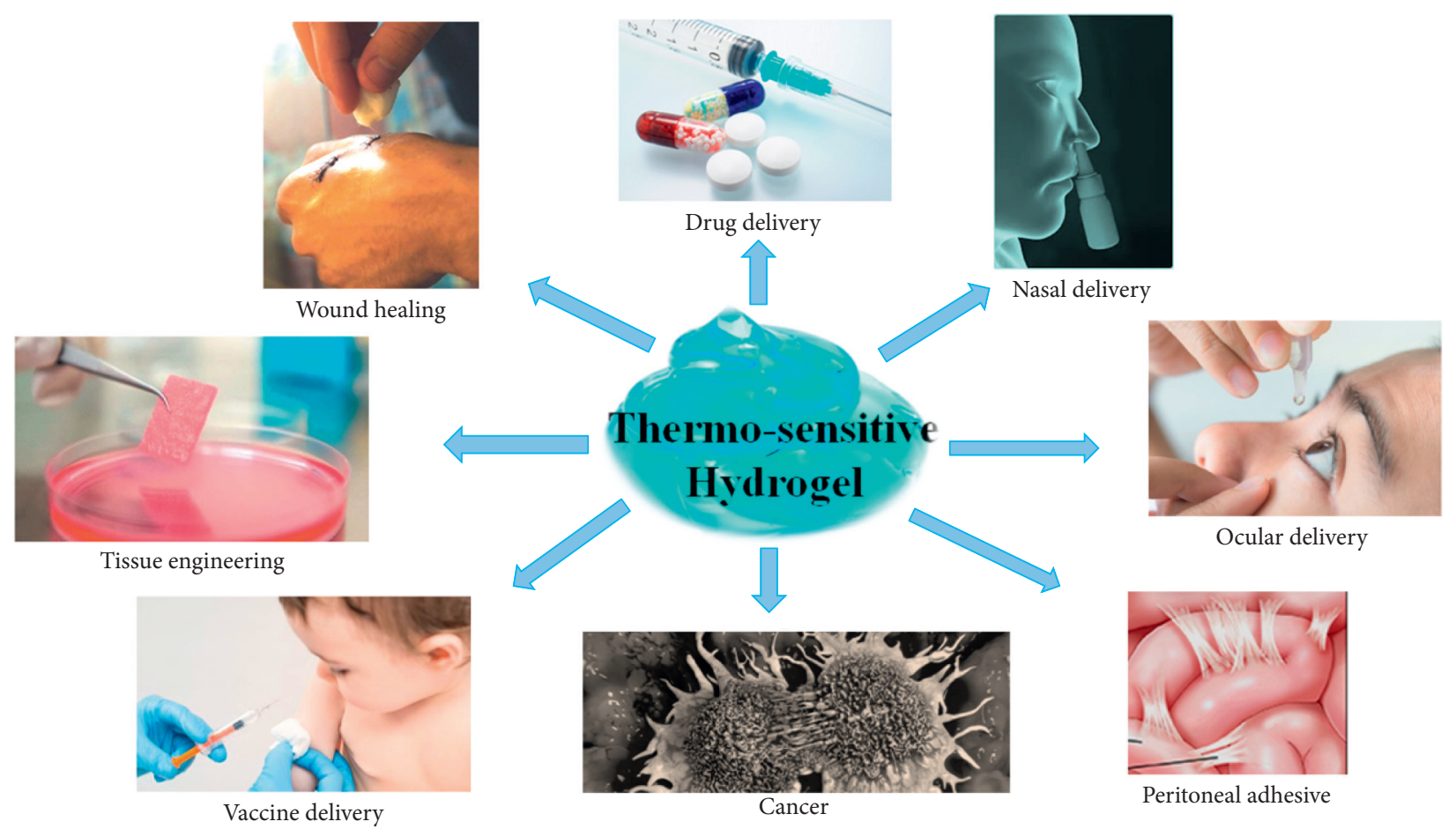

FIgURE 3: Applications of thermosensitive hydrogel.

chitosan- $\beta$-glycerophosphate hydrogels retain the primary polymer's critical properties but enhance its solubility, adhesiveness, and temperature-sensitive properties, and activate the product at a broader range of $\mathrm{pH}[57,106]$. A nasal formulation should be formulated to be liquid at room temperature and also used via spray. It becomes a gel at $32-35^{\circ} \mathrm{C}$ from the liquid state and has good rheological and adhesive properties for remaining in the nasal mucosa for a long time. In one study, hydrogel formulations were prepared from the combination of polyethene glycol and glycerophosphate with n-tri-methyl chitosan, which was gelled at $32.5^{\circ} \mathrm{C}$ for 7 minutes. This hydrogel provided good rheological properties to the mucosa and was well placed on the mucosal surfaces. It seems that this formulation could be a good choice for nasal drug delivery [107].

Temperature-sensitive hydrogels are an ideal system for delivering vaccines through the nose. These systems are used to transport fragmented $\mathrm{H} 5 \mathrm{~N} 1$ and Ebola antigens. This system disperses the vaccine well into the nasal mucosa, holds the antigen on the nasal mucosa for a long time, and opens tight junctions of the nasal epithelial cells, thereby increasing vaccine uptake. Therefore, chitosan- $\beta$-glycerophosphate hydrogels can also be used for vaccine delivery [108].

6.1.2. Ocular Delivery. In a study in 2015, the effect of chitosan hydrogel containing ferulic acid on burn injury in the cornea was assessed. Topical use of chitosan-ferulic acid hydrogel drops improved the rate of corneal healing compared to the control group. It was also observed less hyperplasia on the corneal surface in the group treated with a Hyperplasia was also lower on the corneal surface in hydrogel containing ferulic acid group $(\mathrm{pH}=7.4)$ [76].
Moreover, one study in 2016 represented that chitosangelatine-glycerolphosphate hydrogels containing latanoprost could significantly reduce the intraocular pressure following the triamcinolone acetone-induced glaucoma in the rabbit model. In this study, a temperature-sensitive hydrogel was designed wherein gel formation occurred at $34.18^{\circ} \mathrm{C}$ and provided a stable release profile. Due to its adhesive mucosal properties, chitosan has long been attached to the eye's mucosa and provides a longer release profile. After one week, the intraocular pressure was significantly reduced compared to the control group (one drop per week). Finally, it was observed that the pressure in the eyes was within a normal range. This formulation was converted to gelling at $34.18^{\circ} \mathrm{C}$, initially assumed to be below the eye temperature, but it was observed that the formulation became gel in approximately 70 seconds. In this study, the deacetylation rate of chitosan was more than 95\% [109].

6.1.3. Lung Delivery. Chitosan- $\beta$-glycerophosphate hydrogels have been studied in the treatment of pulmonary emphysema. In emphysema, the elasticity and structure of the alveoli are lost, resulting in airway collapse. There is currently no definitive treatment, and it is only possible to slow down its progression and treat its symptoms. In patients with severe emphysema, we have surgery or a lung transplant. Using hydrogels, we can use it as a factor to seal the damaged area. Chitosan hydrogel is used in this case due to its biocompatibility and degradability. We can easily transfer the gel to the target site in the lungs, and by injecting it at the site, it fills the damaged areas. The three-dimensional network structure of the hydrogel and its physical properties, such as stability and adhesiveness, and the high gel rate, 
provide beneficial characteristics. In one study, chitosan $\beta$-glycerophosphate hydrogel was used as a lung sealant for dogs with biological lung volume reduction (BLVR). The hydrogel is liquefied at room temperature. It is easily injected into the site of injury and becomes a gel, and remains after a few minutes on the site. After three weeks, it was observed that the chitosan $\beta$-glycerophosphate hydrogel created low stability and mechanical strength at the site. Thus, a certain percentage of genipine was added as a nontoxic cross-linker, with outstanding results [110].

6.1.4. Vaginal Delivery. Pseudoplastic semisolid formulations are considered more suitable for vaginal administration because they offer increased flow, which helps in extrusion from the applicator and enables avoidance of leakage from the administration site. Chitosan can contact and attach the vaginal surface well due to its sticky mucus properties. One of the challenges we face about chitosan hydrogel is the sudden initial release of the drug. In one study, the drug was placed in a microsphere and loaded into a hydrogel. This procedure significantly reduced the initial and sudden release rate. Mucosal hydrogels improve drug targeting in the vaginal cavity due to prolonged exposure to the drug at the site of infection or inflammation. The parameters that affect the hydrogel properties of vaginal administration are [111] as follows: (1) Cohesiveness: the higher the cohesiveness of the hydrogel, the better because it regenerates faster and forms a gel, improving product performance. (2) Hardness: if the hardness is low, the hydrogel is easily removed from the prescription site, but if it is high, it stays in place longer, and the drug is released for a long time. (3) Inflation: this property depends on the environment's $\mathrm{pH}$, the ions inside the environment, the active groups on the polymer, and the polymer bonds, which affect the speed of drug release. (4) Mucosal adhesive properties: mucosal adhesive properties are the bonds between hydrogel chains and mucosal surfaces. Chitosan $\beta$-Glycerophosphate hydrogels are adhesive due to the presence of chitosan in the mucosal property [111].

One of the convenient features of chitosan hydrogel is the mucosal adhesive properties and anticandidiasis effects of chitosan. One of the most important features in vaginal delivery systems is that the carriers should be stable in the $\mathrm{pH}$ of this area and should not change the physiological $\mathrm{pH}$, which leads to fungal and bacterial infections. Chitosan hydrogel provides a uniform release of the drug into the vagina, but it increases the $\mathrm{pH}$ of the site. This uniform release is caused by high water absorption and numerous pores. In general, chitosan $\beta$-glycerophosphate hydrogels can be a suitable option for vaginal delivery [111].

6.2. Diabetes (Insulin). The chitosan- $\beta$-glycerophosphate solution provides a constant $\mathrm{pH}$ range in the physiological range (6.6-6.8) that is not changed during the gelation. In one study, the insulin release from chitosan- $\beta$-glycerophosphate hydrogels was investigated. It was observed that insulin is released from hydrogels within two weeks. As the concentration of $\beta$-glycerophosphate increased, the amount of secreted insulin and its release rate also increased. This formulation contained $2.5 \%$ chitosan and $8.16 \% \beta$-glycerophosphate. In this study, it was observed that at least $6 \%$ $\beta$-glycerophosphate must be present in the formulation for carrying out the gelation process. In this study investigating the thermosensitive chitosan hydrogels containing insulin, chitosan- $\beta$-glycerophosphate hydrogel maintains insulin stability under physiological conditions and can be used as a formulation for the delivery of peptide and protein drugs. They placed different concentrations of insulin in two solutions containing different concentrations of chitosanglycerophosphate and examined the drug release in vivo over three weeks. The results showed that drug release decreased with increasing $\beta$-glycerophosphate concentration from the formulation. An 8-amino-1-naphthalene sulfonate probe evaluated the stability of the released insulin. The results showed that its natural structure releases insulin. Eventually, the study showed that chitosan hydrogels containing $\beta$-glycerophosphate could be used to transfer insulin to the body $[10,53]$.

6.3. Cancer. In one study, it has been shown that administration of a thermosensitive chitosan hydrogel containing paclitaxel on EMT-6 murine mammary cancer cells, equivalent to four intravenous injections of this drug, inhibited cell growth. The results show that chitosan$\beta$-glycerophosphate hydrogel can control cancer cells' growth even in the absence of the drug. One of these causes may be due to chitosan's antitumour effect and its derivatives inhibiting cancer cells' growth [15]. In one study, chitosan- $\beta$-glycerophosphate hydrogels containing paclitaxel were prepared. In this study, to increase the solubility of the drug, the drug was first loaded on cyclodextrin, and then to improve the physicochemical properties of the hydrogel, polyvinyl alcohol (PVA) was added to the hydrogel. Studies have shown that $\operatorname{Taxol}^{\circledR}$ formulation completely releases the drug during the 24 hours, while hydrogel formulation can release $82 \%$ of the drug during the 13 days. It was found that intratumour administration of paclitaxel hydrogels had approximately 3 -fold more significant antitumour effects than the commercial formulation of paclitaxel $\left(\operatorname{Taxol}^{\circledR}\right)$ [112].

6.4. Hydatid Cyst. There are several methods for the treatment of hydatid cysts, from surgery to chemotherapy. The most important concern is about injury to the cyst during surgery. If the cyst ruptured during surgery, the material that comes out can cause new cysts to form on the site and cause anaphylactic shock or even death. If we can somehow solidify the fluid inside the cyst, the risk might be reduced. Injectable, temperature-sensitive hydrogels can be a good choice as a gelating agent. In one study, chitosan $\beta$-glycerophosphate hydrogels were used to control cyst subsidence during aspiration. They swell in aquatic environments and have a high capacity to absorb water or physiological solutions in their network structure. The amount of fluid absorbed in hydrogels depends on the formulation, impurity, and salt content. The reason for choosing this type of gel 
strength hydrogel is the uniform internal network. The hydrogel solution was injected into the cyst with an insulin syringe, and after a particular time, it became a gel. The gel duration is related to the physicochemical nature of the cyst fluid. The concentration of salt and the type of salt ion can reduce the gelating time. After gelation, the cyst can be removed more easily from its place because the possibility of rupture is reduced [113].

6.5. Spinal Cord Injury. Chitosan $\beta$-glycerophosphate hydrogels can be used as a carrier to transfer cells to the site of injury. In one study, mesenchymal stem cells were loaded into hydrogels. The paracrine function of mesenchymal stem cells improves, repairs, and reduces cell death in the spinal cord. Due to its high biocompatibility and anti-inflammatory and antioxidant effects, chitosan $\beta$-glycerophosphate hydrogels are an excellent option for mesenchymal stem survival cells. Mesenchymal stem cells secrete a wide range of growth factors, cytokines, and extracellular vesicles, which regulate cell-to-cell and cell-to-extracellular matrix functions. Chitosan $\beta$-glycerophosphate hydrogels contain large amounts of water and a viscoelastic surface close to living tissue that can simulate the spinal cord's mechanical properties. In this study, it was observed that the mesenchymal stem cells transferred to the damaged spinal cord were able to reduce the volume of the lesion and improve spinal function. However, the study found that the number of mesenchymal stem cells that remained in place after recovery had decreased due to poor environmental conditions at the injury site. Chitosan substrates induced the differentiation of cortical and spinal cord progenitor cells into cortical neurons and motor neurons and promoted active synaptic networks' rearrangement [114].

6.6. Wound Healing. Many factors, such as molecular weight, flexibility, cross-linking density, and hydrogen-bonding capacity, affect chitosan hydrogels' adhesive properties. The high molecular weight of chitosan $(\sim 340 \mathrm{kDa})$ and gelatine can improve the adhesion properties of hydrogen adhesives. Both hydrogel and gelatine molecules have many hydrophilic groups that form hydrogen bonds between them and increase their mucosal adhesive properties and increase the drug release duration [103]. Chitosan is a cationic polymer that can bind to and attach to mucin's negative charge, thus providing excellent mucosal adhesive properties [115]. One of the challenges we are faced with chitosan hydrogels is the sudden initial release of the drug [116]. In a study of chitosan membranes used for wounds healing, it has been observed that the scar formation of burns on the rabbit cornea due to the alkaline burns model is reduced [100].

In another study, vancomycin hydrochloride microparticles were first loaded into the HPMC by the spray dryer method. These particles were found to have an average diameter of $1.5-6.4 \mu \mathrm{m}$, had a good dispersion, and made approximately homogeneous particles. The drug was encapsulated at about $72.6 \%$. The drug was then added to the chitosan- $\beta$-glycerophosphate solution to give a slow-release formulation. The in vitro results showed good release kinetics, and the drug was released at a reasonable rate for a long time from the hydrogel [25]. In general, this formulation can release the drug appropriately and prevent wound infection. Studies show that hydrogel is a very suitable formulation for the treatment of chronically infected wounds. However, it has problems such as the high initial release of the drug. In another study, a new formulation of cefuroxime was developed that slowly releases and prolongs the drug's release as a hydrogel matrix made of chitosan. In this study, different concentrations of cefuroxime were loaded in hydrogels. It has been found that phosphate buffer ( $\mathrm{pH}$ 7.4) released more drugs than the medium containing stearate and alkaline $(\mathrm{pH}=10)$. Studies on the L929 fibroblast and MG-63 osteosarcoma cell lines showed that this hydrogel was biocompatible and could be used for long-term drug delivery in chronic wounds [117].

6.7. Cell Therapy/Tissue Engineering. Injectable hydrogels can cause irregular shapes, survive for long in the tissue, and do not stimulate the immune system, so that they can be an appropriate candidate for cell therapy [118]. The hydrogels' porous structure improves drug release into the environment without altering the physical and chemical structure of the drug [119]. The cell encapsulated within the hydrogel can easily exchange materials with the surrounding environment, receive various factors, divide and grow up [120]. Many hydrogels are created using either organic compounds or organic binders that negatively affect cellular activity [96]. Hydrogels can support cells during tissue regeneration and drug delivery systems because the hydrogel's physical and chemical properties are similar to the body's natural extracellular matrix. Tissue engineering (TE) is a promising method for repairing damaged bone tissue. Injectable hydrogels are positioned through a single injection using a wide range of materials used as fillers without intervention and surgery and are used in applications such as bone defect improvement. Chitosan hydrogel has several benefits, including similarity to the extracellular matrix (ECM) and providing a unique microenvironment for cell growth that can be of great use in cell culture [121]. Hydrogels have a high viscosity and high ability to eject the syringe at the time of injection. After ejection from the needle and presence in the environment, their viscosities increased rapidly and became a gel and hardened. The best feature of these hydrogels is their high resistance to internal stresses, making them suitable for cell therapy and tissue engineering.

The following are some of the limitations of cell therapy: $\mathrm{p}$ (a) the difficulty in producing an adequate combination of gelation kinetics and cytocompatibility, (b) their inferior mechanical properties after gelation, (c) poor survival and weak cell proliferation (d) toxicity of beta-glycerophosphate at a concentration above $0.12 \mathrm{M}$ for cells also cause hyperosmolarity of the hydrogel. To eliminate these limitations, we can add other compounds such as dibasic ammonium hydrogen phosphate, dibasic hydrogen phosphate, or sodium hydrogen carbonate to the hydrogel structure or replace it with $\beta$-glycerophosphate. Due to the body's physiological pH and the gel's osmolality, cell survival is well 
preserved. Adequate studies on the presence of pores in the gel and its relationship with cell survival or oxygen diffusion to hydrogel are not available, and further research is required. Studies have shown that chitosan increases the cell viability and distribution capacity of encapsulated fibroblasts in serum and plasma environments. The ability of the hydrogel to absorb the water causes the exchange of nutritious material and ions required by the cell with the surrounding environment. Therefore, it is a suitable environment for the growth of cells and tissues. Hydrogels can create an elastic environment similarly to body tissues. Creating this elasticity and compressive strength by using a biodegradable hydrogel without creating new chemical bonds is an advantage of chitosan. Various studies show that these hydrogels can regenerate soft tissues and even tissues such as cartilage under high pressure [122].

6.8. Peritoneal Adhesion. Peritoneal adhesion is considered a significant problem that occurs after peritoneal surgery. In one-third of surgeries, the connective tissue is created between the injured areas, which can cause many problems, including bowel obstruction. Occasionally, after surgeries, such as childbirth, very large adhesions can develop that they can also involve the ovary and cause complete infertility $[123,124]$. In a study performed in 2006, chitosan-containing gels were found to have anti-adhesion effects on tissue damage to the peritoneum but did not affect external adhesion factors, such as talc. The chitosan film created in the injured area remains in place for more than two weeks, which can exacerbate its adhesion. The addition of gelatine to chitosan causes the film to be more biodegradable in less time and reduces adhesion probability $[104,105]$. In another study, naproxen (a hydrophobic drug) nanoparticles were embedded in chitosan- $\beta$-glycerophosphate hydrogels. In this study, a rat model was used to induce adhesion and test the formulation. At the end of the study, the hydrogel was easily separated from the peritoneal wall. It has been observed that on the 7th day after surgery, the wounds created during surgery were entirely covered by an epithelial layer; however, lesions were attached and provided severe adhesion in the negative control group. Also, chitosan hydrogel containing naproxen had fewer toxic effects on central tissues and organs, including the liver, spleen, heart, lung, and kidney [106].

\section{Conclusion}

The chitosan- $\beta$-glycerophosphate hydrogel is an intelligent drug system widely used to deliver hydrophilic and hydrophobic drugs to different parts of the body. It can store a large amount of drug in its three-dimensional network and release it to the environment at a controlled rate at a specific location. Changes in this system's components, such as changes in chitosan concentration, the molecular weight of chitosan, degree of acetylation, glycerophosphate concentration, and the addition of other reagents, can control the formation gel's time, temperature, and $\mathrm{pH}$. Moreover, by increasing glycerophosphate salt concentration, the concentration of chitosan solution, and DDA or ambient temperature, chitosan solutions' gelling time can be reduced. Due to its good features, such as being biodegradable and biocompatible with the human body and the ability to stay in place for a long time and controlled drug release, this drug delivery system can be an excellent option to replace other drug forms of implants. Also, due to its three-dimensional and highly porous structure, this system can provide a suitable environment for the growth and proliferation of a wide range of cells, which will be an appropriate option in the field of tissue engineering. Besides, by adding other compounds, such as gelatine, polyvinyl alcohol, and graphene oxide, to Chitosan-b glycerophosphate hydrogel, changes in the drug release rate and gel formation time can be made. These new systems are more efficient than chitosan beta-glycerophosphate hydrogels. Due to this drug delivery system's desirable features, it is predicted that the drug-containing formulations from this drug system will enter the global market in the future.

\section{Abbreviations}

LCST: $\quad$ Lower critical solution temperature

UCST: Upper critical solution temperature

PEO: $\quad$ Poly (ethylene oxide)

PPO: $\quad$ Polyphenylene oxide (PPO)

PEG: $\quad$ Poly (ethylene glycol)

PVA: $\quad$ Poly (vinyl alcohol)

DHO: Dipotassium hydrogen orthophosphate

OS: Osteosarcoma

FDA: $\quad$ Food and Drug Administration

TPP: Anionic triphosphate groups

TEM: Transmission electron microscopy

GP: $\quad$ Glycerophosphate

DDA: $\quad$ Degree of deacetylation

$\mathrm{NaHCO}_{3}$ : $\quad$ Sodium hydrogen carbonate

Chitosan- N-[(2-hydroxy-3-trimethylammonium)

HTICC: $\quad$ propyl] chitosan chloride

MSC: $\quad$ Mesenchymal stem cell

LSCM: Laser scanning confocal microscopy

PLGA: $\quad$ Poly (lactic-co-glycolic acid)

kD: $\quad$ Kilo Dalton

MW: Molecular weight

SEM: $\quad$ Scanning electron microscope

CS: Chitosan

TE: Tissue engineering

ECM: $\quad$ Extracellular matrix

-G-GP: $\quad \beta$-Glycerophosphate

HPMC: $\quad$ Hydroxypropyl methylcellulose

5-FU: 5-Fluorouracil.

\section{Data Availability}

Data will be available on request to the corresponding author.

\section{Conflicts of Interest}

The authors declare no conflicts of interest. 


\section{Acknowledgments}

This study was financially supported by a nonspecific grant from the Mashhad University of Medical Sciences' Research Council.

\section{References}

[1] S. Supper, N. Anton, N. Seidel, M. Riemenschnitter, C. Curdy, and T. Vandamme, "Thermosensitive chitosan/ glycerophosphate-based hydrogel and its derivatives in pharmaceutical and biomedical applications," Expert Opinion on Drug Delivery, vol. 11, no. 2, pp. 249-267, 2014.

[2] M. I. H. Mondal, Cellulose-based Superabsorbent Hydrogels, Springer, Berlin, Germany, 2019.

[3] S. Khoee and K. Kardani, "Hydrogels as controlled drug delivery carriers," Polymerization, vol. 2, no. 4, pp. 16-27, 2013.

[4] R. Chang and J. R. Robinson, "Sustained release from tablets and particles through coating," Pharmaceuticals Dosage Form Tablets, vol. 3, pp. 199-302, 1990.

[5] B. Amsden and B. Amsden, "Solute diffusion in hydrogels," Polymer Gels and Networks, vol. 6, no. 1, pp. 13-43, 1998.

[6] (Gupta, Vermani, and Garg 2002, Mathew et al. 2018).

[7] A. R. Kharel and N. A. Peppas, "Preparation, structure and diffusional behavior of hydrogels in controlled release," Advanced Drug Delivery Reviews, vol. 11, pp. 1-35, 1993.

[8] E. M. Ahmed, "Hydrogel: preparation, characterization, and applications: a review," Journal of Advanced Research, vol. 6, no. 2, pp. 105-121, 2015.

[9] S. V. Vinogradov, E. V. Batrakova, and A. V. Kabanov, "Nanogels for oligonucleotide delivery to the brain," Bioconjugate Chemistry, vol. 15, no. 1, pp. 50-60, 2004.

[10] S. Kempe, H. Metz, M. Bastrop, A. Hvilsom, R. V. Contri, and K. Mäder, "Characterization of thermosensitive chitosan-based hydrogels by rheology and electron paramagnetic resonance spectroscopy," European Journal of Pharmaceutics and Biopharmaceutics, vol. 68, no. 1, pp. 26-33, 2008.

[11] B. M. Jeong, S. W. Kim, and Y. H. Baeb, "Thermosensitive sol-gel reversible hydrogels," Advanced Drug Delivery Reviews, vol. 64, pp. 154-162, 2012.

[12] S. R. M. Montoro and G. M. Alves, "Nanostructured hydrogels," 2013.

[13] P. Pankongadisak and O. Suwantong, "Enhanced properties of injectable chitosan-based thermogelling hydrogels by silk fibroin and longan seed extract for bone tissue engineering," International Journal of Biological Macromolecules, vol. 138, pp. 412-424, 2019.

[14] L. Li, H. Shan, C. Y. Yue, Y. C. Lam, K. C. Tam, and X. Hu, "Thermally induced association and dissociation of methylcellulose in aqueous solutions," Langmuir, vol. 18, no. 20, pp. 7291-7298, 2002.

[15] E. Ruel-Gariépy and J.-C. Leroux, "In situ-forming hydrogels-review of temperature-sensitive systems," European Journal of Pharmaceutics and Biopharmaceutics, vol. 58, no. 2, pp. 409-426, 2004.

[16] J. H. Cho, S.-H. Kim, K. D. Park et al., "Chondrogenic differentiation of human mesenchymal stem cells using a thermosensitive poly(N-isopropylacrylamide) and watersoluble chitosan copolymer," Biomaterials, vol. 25, no. 26, pp. 5743-5751, 2004.

[17] A. Chenite, C. Chaput, D. Wang et al., "Novel injectable neutral solutions of chitosan form biodegradable gels in situ," Biomaterials, vol. 21, no. 21, pp. 2155-2161, 2000.
[18] M. R. Steinwachs, B. Waibl, and M. Mumme, "Arthroscopic treatment of cartilage lesions with microfracture and BSTCarGel," Arthroscopy Techniques, vol. 3, no. 3, pp. e399-e402, 2014.

[19] X. Zhang, D. Wu, and C.-C. Chu, "Synthesis and characterization of partially biodegradable, temperature and $\mathrm{pH}$ sensitive Dex-MA/PNIPAAm hydrogels," Biomaterials, vol. 25, no. 19, pp. 4719-4730, 2004.

[20] H. Yang and W. J. Kao, "Thermoresponsive gelatin/monomethoxy poly(ethylene glycol)-poly(d,l-lactide) hydrogels: formulation, characterization, and antibacterial drug delivery," Pharmaceutical Research, vol. 23, no. 1, pp. 205-214, 2006.

[21] C. Joly-Duhamel, D. Hellio, and M. Djabourov, "All gelatin networks: 1. Biodiversity and physical chemistry," Langmuir, vol. 18, no. 19, pp. 7208-7217, 2002.

[22] L. Klouda and A. G. Mikos, "Thermoresponsive hydrogels in biomedical applications," European Journal of Pharmaceutics and Biopharmaceutics, vol. 68, no. 1, pp. 34-45, 2008.

[23] J. Shalhoub, R. J. Hinchliffe, and J. T. Powell, "The world of legoo assessed: a short systematic and critical review," European Journal of Vascular and Endovascular Surgery, vol. 45, no. 1, pp. 44-45, 2013.

[24] A. V. Kabanov, E. V. Batrakova, and V. Y. Alakhov, "Pluronic ${ }^{\circledR}$ block copolymers as novel polymer therapeutics for drug and gene delivery," Journal of Controlled Release, vol. 82, no. 2-3, pp. 189-212, 2002.

[25] A. Steinleitner, H. Lambert, C. Kazensky, and B. Cantor, "Poloxamer 407 as an intraperitoneal barrier material for the prevention of postsurgical adhesion formation and reformation in rodent models for reproductive surgery," $\mathrm{Ob}$ stetrics and Gynecology, vol. 77, no. 1, pp. 48-52, 1991.

[26] C. Gong, T. Qi, X. Wei et al., "Thermosensitive polymeric hydrogels as drug delivery systems," Current Medicinal Chemistry, vol. 20, no. 1, pp. 79-94, 2013.

[27] N. Bhattarai, J. Gunn, and M. Zhang, "Chitosan-based hydrogels for controlled, localized drug delivery," Advanced Drug Delivery Reviews, vol. 62, no. 1, pp. 83-99, 2010.

[28] N. Bhattarai, F. A. Matsen, and M. Zhang, "PEG-grafted chitosan as an injectable thermoreversible hydrogel," Macromolecular Bioscience, vol. 5, no. 2, pp. 107-111, 2005.

[29] J. M. Dang, D. D. N. Sun, Y. Shin-Ya, A. N. Sieber, J. P. Kostuik, and K. W. Leong, “Temperature-responsive hydroxybutyl chitosan for the culture of mesenchymal stem cells and intervertebral disk cells," Biomaterials, vol. 27, no. 3, pp. 406-418, 2006.

[30] S. Chatterjee, P. C. Hui, and C. W. Kan, "Thermoresponsive hydrogels and their biomedical applications: special insight into their applications in textile based transdermal therapy," Polymers (Basel), vol. 10, no. 5, 2018.

[31] Y. Tang, Y. Du, Y. Li, X. Wang, and X. Hu, "A thermosensitive chitosan/poly(vinyl alcohol) hydrogel containing hydroxyapatite for protein delivery," Journal of Biomedical Materials Research Part A, vol. 91A, no. 4, pp. 953-963, 2009.

[32] H. T. Ta, H. Han, I. Larson et al., "Chitosan-dibasic orthophosphate hydrogel: a potential drug delivery system," International Journal of Pharmaceutics, vol. 371, no. 1-2, pp. 134-141, 2009.

[33] Y.-Y. Liu, Y.-H. Shao, and J. Lü, "Preparation, properties and controlled release behaviors of $\mathrm{pH}$-induced thermosensitive amphiphilic gels," Biomaterials, vol. 27, no. 21, pp. 4016-4024, 2006.

[34] Y. Qiu and K. Park, "Environment-sensitive hydrogels for drug delivery," Advanced Drug Delivery Reviews, vol. 53, no. 3, pp. 321-339, 2001. 
[35] A. S. Hoffman, "Hydrogels for biomedical applications," Advanced Drug Delivery Reviews, vol. 54, no. 1, pp. 3-12, 2002.

[36] M. A. Mohammed, "An overview of chitosan nanoparticles and its application in non-parenteral drug delivery," Pharmaceutics, vol. 9, no. 4, 2017.

[37] S. Bagheri-khoulenjai, H. Mirzadeh, and M. Etrati-Khosroshahi, "Chitosan and nanohydroxyapatite roles in physical and chemical characteristics of gelatin/chitosan/nanohydroxyapatite microspheres," Iranian Journal of Polymer Science and Technology, vol. 23, no. 6, pp. 487-498, 2011.

[38] J. Wu, W. Wei, L.-Y. Wang, Z.-G. Su, and G.-H. Ma, "A thermosensitive hydrogel based on quaternized chitosan and poly(ethylene glycol) for nasal drug delivery system," Biomaterials, vol. 28 , no. 13, pp. 2220-2232, 2007.

[39] S. S. Koide, "Chitin-chitosan: properties, benefits and risks," Nutrition Research, vol. 18, no. 6, pp. 1091-1101, 1998.

[40] M. N. V. R. Kumar, R. A. A. Muzzarelli, C. Muzzarelli, H. Sashiwa, and A. J. Domb, "Chitosan chemistry and pharmaceutical perspectives," Chemical Reviews, vol. 104, no. 12, pp. 6017-6084, 2004.

[41] O. Felt, P. Buri, and R. Gurny, "Chitosan: a unique polysaccharide for drug delivery," Drug Development and Industrial Pharmacy, vol. 24, no. 11, pp. 979-993, 1998.

[42] C. Chatelet, O. Damour, and A. Domard, "Influence of the degree of acetylation on some biological properties of chitosan films," Biomaterials, vol. 22, no. 3, pp. 261-268, 2001.

[43] S. Miyazaki, K. Ishii, and T. Nadai, "The use of chitin and chitosan as drug carriers," Chemical and Pharmaceutical Bulletin, vol. 29, no. 10, pp. 3067-3069, 1981.

[44] S. Mansouri, P. Lavigne, K. Corsi, M. Benderdour, E. Beaumont, and J. C. Fernandes, "Chitosan-DNA nanoparticles as non-viral vectors in gene therapy: strategies to improve transfection efficacy," European Journal of Pharmaceutics and Biopharmaceutics, vol. 57, no. 1, pp. 1-8, 2004.

[45] R. J. Nordtveit, K. M. Vårum, and O. Smidsrød, "Degradation of fully water-soluble, partially $\mathrm{N}$-acetylated chitosans with lysozyme," Carbohydrate Polymers, vol. 23, no. 4, pp. 253-260, 1994.

[46] Y. Ohya, M. Shiratani, H. Kobayashi, and T. Ouchi, "Release behavior of 5-fluorouracil from chitosan-gel nanospheres immobilizing 5-fluorouracil coated with polysaccharides and their cell specific cytotoxicity," Journal of Macromolecular Science, Part A, vol. 31, no. 5, pp. 629-642, 1994.

[47] M. Hamidi, A. Azadi, and P. Rafiei, "Hydrogel nanoparticles in drug delivery," Advanced Drug Delivery Reviews, vol. 60, no. 15, pp. 1638-1649, 2008.

[48] R. Fernandez-Urrusuno, "Enhancement of nasal absorption of insulin using chitosan nanoparticles," Pharmaceutical Research, vol. 16, no. 10, pp. 1576-1581, 1999.

[49] L. Zhou, K. Men, S. Zeng et al., "Codelivery of SH-aspirin and curcumin by mPEG-PLGA nanoparticles enhanced antitumor activity by inducing mitochondrial apoptosis," International Journal of Nanomedicine, vol. 10, pp. 52055218, 2015.

[50] Y. Xu and Y. Du, "Effect of molecular structure of chitosan on protein delivery properties of chitosan nanoparticles," International Journal of Pharmaceutics, vol. 250, no. 1, pp. 215-226, 2003.

[51] K. M. Picker-Freyer and D. Brink, "Evaluation of powder and tableting properties of chitosan," AAPS PharmSciTech, vol. 7, no. 3, p. 75, 2006.

[52] K. A. Janes, P. Calvo, and M. J. Alonso, "Polysaccharide colloidal particles as delivery systems for macromolecules,"
Advanced Drug Delivery Reviews, vol. 47, no. 1, pp. 83-97, 2001.

[53] E. Khodaverdi, M. Tafaghodi, F. Ganji, K. Abnoos, and H. Naghizadeh, "In vitro insulin release from thermosensitive chitosan hydrogel," AAPS PharmSciTech, vol. 13, no. 2, pp. 460-466, 2012.

[54] F. Ganji, "In vitro sustained release of pyridostigmin bromide from injectable thermosensitive chitosan hydrogel," Iranian Journal of Biomedical Engineering, vol. 8, no. 3, pp. 249-260, 2014.

[55] F. Langenbach and J. Handschel, "Effects of dexamethasone, ascorbic acid and $\beta$-glycerophosphate on the osteogenic differentiation of stem cells in vitro," Stem Cell Research \& Therapy, vol. 4, no. 5, p. 117, 2013.

[56] S. Ladet, L. David, and A. Domard, "Multi-membrane hydrogels," Nature, vol. 452, no. 7183, pp. 76-79, 2008.

[57] J. Wu, Z. G. Su, and G. H. Ma, "A thermo- and pH-sensitive hydrogel composed of quaternized chitosan/glycerophosphate," International Journal of Pharmaceutics, vol. 315, no. 1-2, pp. 1-11, 2006.

[58] K. E. Crompton, J. D. Goud, R. V. Bellamkonda et al., "Polylysine-functionalised thermoresponsive chitosan hydrogel for neural tissue engineering," Biomaterials, vol. 28, no. 3, pp. 441-449, 2007.

[59] M. M. Aliaghaei, H. Mirzadeh, E. Dashtimoghadam, and S. Taranejoo, "Investigation of gelation mechanism of an injectable hydrogel based on chitosan by rheological measurements for a drug delivery application," Soft Matter, vol. 8, no. 27, pp. 7128-7137, 2012.

[60] E. Ruel-Gariepy, "Characterization of thermosensitive chitosan gels for the sustained delivery of drugs," International Journal of Pharmaceutics, vol. 203, no. 1-2, pp. 89-98, 2000.

[61] Y.-M. Tsai, W.-C. Jan, C.-F. Chien, W.-C. Lee, L.-C. Lin, and T.-H. Tsai, "Optimised nano-formulation on the bioavailability of hydrophobic polyphenol, curcumin, in freely-moving rats," Food Chemistry, vol. 127, no. 3, pp. 918-925, 2011.

[62] M. D. A. Azadi, S. Hassanajili, K. Zarrabi, and B. Sarkari, "Correction to: solidification of hydatid cyst fluid with an injectable chitosan/carboxymethylcellulose/ $\beta$-glycerophosphate hydrogel for effective control of spillage during aspiration of hydatid cysts," Progress in Biomaterials, vol. 7, no. 2, p. 151, 2018.

[63] X. Liu, Y. Chen, Q. Huang, W. He, Q. Feng, and B. Yu, “A novel thermo-sensitive hydrogel based on thiolated chitosan/ hydroxyapatite/beta-glycerophosphate," Carbohydrate Polymers, vol. 110, pp. 62-69, 2014.

[64] X. Xu, Z. Gu, X. Chen et al., "An injectable and thermosensitive hydrogel: promoting periodontal regeneration by controlled-release of aspirin and erythropoietin," Acta Biomaterialia, vol. 86, pp. 235-246, 2019.

[65] O. M. Kolawole, W. M. Lau, and V. V. Khutoryanskiy, "Chitosan/ $\beta$-glycerophosphate in situ gelling mucoadhesive systems for intravesical delivery of mitomycin-C," International Journal of Pharmaceutics: X, vol. 1, Article ID 100007, 2019.

[66] Y. Zhao, "Efficacy of thermosensitive chitosan/beta-glycerophosphate hydrogel loaded with beta-cyclodextrin-curcumin for the treatment of cutaneous wound infection in rats," Experimental and Therapeutic Medicine, vol. 15, no. 2, pp. 1304-1313, 2018.

[67] C. Li, S. Ren, Y. Dai et al., "Efficacy, pharmacokinetics, and biodistribution of thermosensitive chitosan/ $\beta$-glycerophosphate hydrogel loaded with docetaxel," AAPS PharmSciTech, vol. 15, no. 2, pp. 417-424, 2014. 
[68] F. Ghasemi Tahrir, F. Ganji, A. R. Mani, and E. Khodaverdi, "In vitro and in vivo evaluation of thermosensitive chitosan hydrogel for sustained release of insulin," Drug Delivery, vol. 23, no. 3, pp. 1038-1046, 2016.

[69] T. Irimia, "Chitosan-based in situ gels for ocular delivery of therapeutics: a state-of-the-art review," Marine Drugs, vol. 16, no. 10, 2018.

[70] X. Kong, W Xu, C Zhang, and W Kong, "Chitosan temperature-sensitive gel loaded with drug microspheres has excellent effectiveness, biocompatibility and safety as an ophthalmic drug delivery system," Experimental and Therapeutic Medicine, vol. 15, no. 2, pp. 1442-1448, 2018.

[71] X. Chao, L. Xu, J. Li et al., "Facilitation of facial nerve regeneration using chitosan- $\beta$-glycerophosphate-nerve growth factor hydrogel," Acta Oto-Laryngologica, vol. 136, no. 6, pp. 585-591, 2016.

[72] Y. Song, N. Nagai, S. Saijo, H. Kaji, M. Nishizawa, and T. Abe, "In situ formation of injectable chitosan-gelatin hydrogels through double crosslinking for sustained intraocular drug delivery," Materials Science and Engineering: $C$, vol. 88, pp. 1-12, 2018.

[73] A. Fabiano, R. Bizzarri, and Y. Zambito, "Thermosensitive hydrogel based on chitosan and its derivatives containing medicated nanoparticles for transcorneal administration of 5-fluorouracil," International Journal of Nanomedicine, vol. 12, pp. 633-643, 2017.

[74] K.-C. Yang, C.-C. Wu, F.-H. Lin et al., "Chitosan/gelatin hydrogel as immunoisolative matrix for injectable bioartificial pancreas," Xenotransplantation, vol. 15, no. 6, pp. 407-416, 2008.

[75] A. Deng, X. Kang, J. Zhang, Y. Yang, and S. Yang, "Enhanced gelation of chitosan/ $\beta$-sodium glycerophosphate thermosensitive hydrogel with sodium bicarbonate and biocompatibility evaluated," Materials Science and Engineering: $C$, vol. 78, pp. 1147-1154, 2017.

[76] A. Naik and H. Nair, "Formulation and evaluation of thermosensitive biogels for nose to brain delivery of doxepin," BioMed Research International, vol. 2014, Article ID 847547, 10 pages, 2014.

[77] T. Kean and M. Thanou, "Biodegradation, biodistribution and toxicity of chitosan," Advanced Drug Delivery Reviews, vol. 62, no. 1, pp. 3-11, 2010.

[78] P. Sacco, F. Furlani, G. de Marzo et al., "Concepts for developing physical gels of chitosan and of chitosan derivatives," Gels, vol. 4, no. 3, 2018.

[79] X. Chen, X. Li, Y. Zhou et al., "Chitosan-based thermosensitive hydrogel as a promising ocular drug delivery system: preparation, characterization, and in vivo evaluation," Journal of Biomaterials Applications, vol. 27, no. 4, pp. 391-402, 2012.

[80] R. Ahmadi and J. D. de Bruijn, "Biocompatibility and gelation of chitosan-glycerol phosphate hydrogels," Journal of Biomedical Materials Research Part A, vol. 86A, no. 3, pp. 824-832, 2008.

[81] K. E. Crompton, R. J. Prankerd, D. M. Paganin et al., "Morphology and gelation of thermosensitive chitosan hydrogels," Biophysical Chemistry, vol. 117, no. 1, pp. 47-53, 2005.

[82] H. Qin, J. Wang, T. Wang et al., "Preparation and characterization of chitosan/beta-glycerophosphate thermal-sensitive hydrogel reinforced by graphene oxide," Frontiers in Chemistry, vol. 6, p. 565, 2018.

[83] E. Ruel-Gariépy, M. Shive, A. Bichara et al., "A thermosensitive chitosan-based hydrogel for the local delivery of paclitaxel," European Journal of Pharmaceutics and Biopharmaceutics, vol. 57, no. 1, pp. 53-63, 2004.

[84] G. Sharma, J. L. Italia, K. Sonaje, K. Tikoo, and M. N. V. Ravi Kumar, "Biodegradable in situ gelling system for subcutaneous administration of ellagic acid and ellagic acid loaded nanoparticles: evaluation of their antioxidant potential against cyclosporine induced nephrotoxicity in rats," Journal of Controlled Release, vol. 118, no. 1, pp. 27-37, 2007.

[85] T. Zhang and D. Herlyn, "Combination of active specific immunotherapy or adoptive antibody or lymphocyte immunotherapy with chemotherapy in the treatment of cancer," Cancer Immunology, Immunotherapy, vol. 58, no. 4, pp. 475-492, 2009.

[86] A. M. Gumel, "Poly (3-hydroxyalkanoates)-co-(6-hydroxyhexanoate) hydrogel promotes angiogenesis and collagen deposition during cutaneous wound healing in rats," Biomedical Materials, vol. 10, no. 4, Article ID 045001, 2015.

[87] R. Galante, C. F. Rediguieri, I. S. Kikuchi et al., "About the sterilization of chitosan hydrogel nanoparticles," PLoS One, vol. 11, no. 12, Article ID e0168862, 2016.

[88] C. Zahraoui and P. Sharrock, "Influence of sterilization on injectable bone biomaterials," Bone, vol. 25, no. 2, pp. 63S-65S, 1999.

[89] C. Jarry, J.-C. Leroux, J. Haeck, and C. Chaput, "Irradiating or autoclaving chitosan/polyol solutions: effect on thermogelling chitosan-.BETA.-glycerophosphate systems," Chemical and Pharmaceutical Bulletin, vol. 50, no. 10, pp. 1335-1340, 2002.

[90] E. Szymanska and K. Winnicka, "Stability of chitosan-a challenge for pharmaceutical and biomedical applications," Marine Drugs, vol. 13, no. 4, pp. 1819-1846, 2015.

[91] S. Khan, K. Patil, N. Bobade, P. Yeole, and R. Gaikwad, "Formulation of intranasal mucoadhesive temperaturemediatedin situgel containing ropinirole and evaluation of brain targeting efficiency in rats," Journal of Drug Targeting, vol. 18, no. 3, pp. 223-234, 2010.

[92] X. Chao, Z. Fan, Y. Han et al., "Effects of local application of methylprednisolone delivered by the C/GP-hydrogel on the recovery of facial nerves," Acta Oto-Laryngologica, vol. 135, no. 11, pp. 1178-1184, 2015.

[93] S.-F. Peng, C.-Y. Lee, M.-J. Hour et al., "Curcumin-loaded nanoparticles enhance apoptotic cell death of U2OS human osteosarcoma cells through the Akt-Bad signaling pathway," International Journal of Oncology, vol. 44, no. 1, pp. 238-246, 2014.

[94] H. Wang, Y. Jia, W. Hu, H. Jiang, J. Zhang, and L. Zhang, "Effect of preparation conditions on the size and encapsulation properties of mPEG-PLGA nanoparticles simultaneously loaded with vincristine sulfate and curcumin," Pharmaceutical Development and Technology, vol. 18, no. 3, pp. 694-700, 2013.

[95] S. Ren, Y. Dai, C. Li et al., "Pharmacokinetics and pharmacodynamics evaluation of a thermosensitive chitosan based hydrogel containing liposomal doxorubicin," European Journal of Pharmaceutical Sciences, vol. 92, pp. 137-145, 2016.

[96] Y.-H. Cheng, S.-H. Yang, W.-Y. Su et al., "Thermosensitive chitosan-gelatin-glycerol phosphate hydrogels as a cell carrier for nucleus pulposus regeneration: an in vitro study," Tissue Engineering Part A, vol. 16, no. 2, pp. 695-703, 2010.

[97] Q. Min, X. Yu, J. Liu et al., "Chitosan-based hydrogels embedded with hyaluronic acid complex nanoparticles for controlled delivery of bone morphogenetic protein-2," Pharmaceutics, vol. 11, no. 5, 2019. 
[98] M. Rezazadeh, V. Akbari, M. Minayian et al., "Effect of freeze drying on stability, thermo-responsive characteristics, and in vivo wound healing of erythropoietin-loaded trimethyl chitosan/glycerophosphate hydrogel," Research in Pharmaceutical Sciences, vol. 13, no. 6, pp. 476-483, 2018.

[99] P. Mura, N. Mennini, C. Nativi, and B. Richichi, "In situ mucoadhesive-thermosensitive liposomal gel as a novel vehicle for nasal extended delivery of opiorphin," European Journal of Pharmaceutics and Biopharmaceutics, vol. 122, pp. 54-61, 2018.

[100] S. Jose, C. R. Ansa, T. A. Cinu et al., "Thermo-sensitive gels containing lorazepam microspheres for intranasal brain targeting," International Journal of Pharmaceutics, vol. 441, no. 1-2, pp. 516-526, 2013.

[101] A. Bernkop-Schnürch and S. Dünnhaupt, "Chitosan-based drug delivery systems," European Journal of Pharmaceutics and Biopharmaceutics, vol. 81, no. 3, pp. 463-469, 2012.

[102] A. Clementino, M. Batger, G. Garrastazu et al., "The nasal delivery of nanoencapsulated statins - an approach for brain delivery," International Journal of Nanomedicine, vol. 11, pp. 6575-6590, 2016.

[103] H. Gholizadeh, S. Cheng, M. Pozzoli et al., "Smart thermosensitive chitosan hydrogel for nasal delivery of ibuprofen to treat neurological disorders," Expert Opinion on Drug Delivery, vol. 16, no. 4, pp. 453-466, 2019.

[104] Y. Sun, L. Zhu, T. Wu, T. Cai, E. M. Gunn, and L. Yu, "Stability of amorphous pharmaceutical solids: crystal growth mechanisms and effect of polymer additives," The AAPS Journal, vol. 14, no. 3, pp. 380-388, 2012.

[105] A. F. Kotzé, H. L. Luessen, B. J. de Leeuw, B. G. de Boer, J. C. Verhoef, and H. E. Junginger, "N-trimethyl chitosan chloride as a potential absorption enhancer across mucosal surfaces: in vitro evaluation in intestinal epithelial cells (Caco-2)," Pharmaceutical Research, vol. 14, no. 9, pp. 1197-1202, 1997.

[106] F. Chen, Z.-R. Zhang, and Y. Huang, "Evaluation and modification of N-trimethyl chitosan chloride nanoparticles as protein carriers," International Journal of Pharmaceutics, vol. 336, no. 1, pp. 166-173, 2007.

[107] H. Nazar, D. G. Fatouros, S. M. van der Merwe et al., "Thermosensitive hydrogels for nasal drug delivery: the formulation and characterisation of systems based on N-trimethyl chitosan chloride," European Journal of Pharmaceutics and Biopharmaceutics, vol. 77, no. 2, pp. 225-232, 2011.

[108] J. Wu, Y.-B. Wu, D.-X. Hao et al., "Research spotlight: thermal-sensitive hydrogels as nasal vaccine delivery systems," Therapeutic Delivery, vol. 3, no. 10, pp. 1151-1153, 2012.

[109] Y.-H. Cheng, T.-H. Tsai, Y.-Y. Jhan et al., “Thermosensitive chitosan-based hydrogel as a topical ocular drug delivery system of latanoprost for glaucoma treatment," Carbohydrate Polymers, vol. 144, pp. 390-399, 2016.

[110] T. Songkroh, H. Xie, W. Yu et al., "In situ forming chitosanbased hydrogel as a lung sealant for biological lung volume reduction," Science Bulletin, vol. 60, no. 2, pp. 235-240, 2015.

[111] E. Szyma $\square$ ska, "The effect of $\beta$-glycerophosphate crosslinking on chitosan cytotoxicity and properties of hydrogels for vaginal application," Polymers, vol. 7, no. 11, pp. 22232244, 2015.

[112] Y. Jiang, X. Meng, Z. Wu, and X. Qi, "Modified chitosan thermosensitive hydrogel enables sustained and efficient anti-tumor therapy via intratumoral injection," Carbohydrate Polymers, vol. 144, pp. 245-253, 2016.
[113] M. D. A. Azadi, S. Hassanjili, K. Zarrabi, and B. Sarkari, "Solidification of hydatid cyst fluid with an injectable chitosan/carboxymethylcellulose/ $\beta$-glycerophosphate hydrogel for effective control of spillage during aspiration of hydatid cysts," Progress in Biomaterials, vol. 7, no. 1, pp. 35-54, 2018.

[114] M. Boido, "Chitosan-based hydrogel to support the paracrine activity of mesenchymal stem cells in spinal cord injury treatment," Scientific Reports, vol. 9, no. 1, pp. 1-16, 2019.

[115] T. Gratieri, G. M. Gelfuso, O. de Freitas, E. M. Rocha, and R. F. V. Lopez, "Enhancing and sustaining the topical ocular delivery of fluconazole using chitosan solution and poloxamer/chitosan in situ forming gel," European Journal of Pharmaceutics and Biopharmaceutics, vol. 79, no. 2, pp. 320-327, 2011.

[116] U. Posadowska, M. Parizek, E. Filova et al., "Injectable nanoparticle-loaded hydrogel system for local delivery of sodium alendronate," International Journal of Pharmaceutics, vol. 485, no. 1-2, pp. 31-40, 2015.

[117] V. Pawar, M. Dhanka, and R. Srivastava, "Cefuroxime conjugated chitosan hydrogel for treatment of wound infections," Colloids and Surfaces B: Biointerfaces, vol. 173, pp. 776-787, 2019.

[118] Z. Bao, C. Jiang, Z. Wang et al., "The influence of solvent formulations on thermosensitive hydroxybutyl chitosan hydrogel as a potential delivery matrix for cell therapy," Carbohydrate Polymers, vol. 170, pp. 80-88, 2017.

[119] D. Seliktar, "Designing cell-compatible hydrogels for biomedical applications," Science, vol. 336, no. 6085, pp. 1124-1128, 2012.

[120] L. Zhao, L. Zhu, F. Liu et al., "pH triggered injectable amphiphilic hydrogel containing doxorubicin and paclitaxel," International Journal of Pharmaceutics, vol. 410, no. 1-2, pp. 83-91, 2011.

[121] S. Saravanan, S. Vimalraj, P. Thanikaivelan, S. Banudevi, and G. Manivasagam, "A review on injectable chitosan/beta glycerophosphate hydrogels for bone tissue regeneration," International Journal of Biological Macromolecules, vol. 121, pp. 38-54, 2019.

[122] C. Ceccaldi, E. Assaad, E. Hui, M. Buccionyte, A. Adoungotchodo, and S. Lerouge, "Optimization of injectable thermosensitive scaffolds with enhanced mechanical properties for cell therapy," Macromolecular Bioscience, vol. 17, no. 6, Article ID 1600435, 2017.

[123] V. R. Askari, V. B. Rahimi, P. Zamani et al., "Evaluation of the effects of Iranian propolis on the severity of post operational-induced peritoneal adhesion in rats," Biomedicine \& Pharmacotherapy, vol. 99, pp. 346-353, 2018.

[124] Y. Roohbakhsh, V. Baradaran Rahimi, S. Silakhori et al., "Evaluation of the effects of peritoneal lavage with rosmarinus officinalis extract against the prevention of postsurgical-induced peritoneal adhesion," Planta Medica, vol. 86, no. 6, pp. 405-414, 2020. 